San Jose State University

SJSU ScholarWorks

Master's Projects

Master's Theses and Graduate Research

Spring 5-22-2017

\title{
Application of Computational Methods to Study the Selection of Authentic and Cryptic Splice Sites
}

Tapomay Dey

San Jose State University

Follow this and additional works at: https://scholarworks.sjsu.edu/etd_projects

Part of the Artificial Intelligence and Robotics Commons, and the Other Computer Sciences Commons

\section{Recommended Citation}

Dey, Tapomay, "Application of Computational Methods to Study the Selection of Authentic and Cryptic Splice Sites" (2017). Master's Projects. 524.

DOI: https://doi.org/10.31979/etd.dqkh-rjd8

https://scholarworks.sjsu.edu/etd_projects/524

This Master's Project is brought to you for free and open access by the Master's Theses and Graduate Research at SJSU ScholarWorks. It has been accepted for inclusion in Master's Projects by an authorized administrator of SJSU ScholarWorks. For more information, please contact scholarworks@sjsu.edu. 


\title{
Application of Computational Methods to Study the Selection of Authentic and Cryptic Splice Sites
}

\author{
A Project \\ Presented to \\ The Faculty of the Department of Computer Science \\ San Jose State University \\ In Partial Fulfillment \\ of the Requirements for the Degree \\ Master of Science
}

By

Tapomay Dey

May 2017 
(C) 2017

Tapomay Dey

ALL RIGHTS RESERVED 


\begin{abstract}
Proteins are building blocks of the bodies of eukaryotes, and the process of synthesizing proteins from DNA is crucial for the good health of an organism [13]. However, some mutations in the DNA may disrupt the selection of 5' or 3' splice sites by a spliceosome. An important research question is whether the disruptions have a stochastic relation to the position of nucleotides in the vicinity of the known authentic and cryptic splice sites. This can be achieved by proving that the authentic and cryptic splice sites are intrinsically different. However, the behavior of the spliceosome is not accurately known. Hence, it is a logical step to model the behavior of the spliceosome using an algorithm that is suitable for modeling unknown functions.

Genetic Algorithms have played an important role in heuristically optimizing NP-Hard search problems [8]. An exhaustive search on the splice site data search space in order to determine the spliceosome function is an NP-Hard problem. Thus, spliceosome function is modeled as a search problem and a Genetic Algorithms based framework is created to prove the hypothesis.

A Random Forest based classifier is proposed to be used as the scoring function. It reduces the rigidity of the comparison mechanism used to compare authentic and cryptic splice sites.
\end{abstract}




\section{ACKNOWLEDGEMENTS}

I would like to thank my advisor, Dr. Sami Khuri, for teaching me many advanced topics in Computer Science and BioInformatics. His consistency in providing timely guidance and unique style of mentoring played a key role in the success of this project.

I would like to thank Dr. Philip Heller and Dr. Thomas Austin for their invaluable inputs and guidance.

I am grateful to Dr. Sharmin Khan, Dr. Katerina Potika, Dr. Mark Stamp, Dr. Jon Pearce, and all my previous mentors. I would also like to thank my family and friends for supporting this venture of mine. 


\section{Contents}

1 Motivation 1

2 Problem Statement 2

3 Data Specifications 3

3.15 'Splice Sites . . . . . . . . . . . . . . . . . . . . . . 3

3.2 Authentic 5' Splice Sites . . . . . . . . . . . . . . . . 3

3.3 Cryptic 5' Splice Sites . . . . . . . . . . . . . . . . 5

3.4 3' Splice Sites . . . . . . . . . . . . . . . . . . . . . 6

3.5 Authentic 3' Splice Sites . . . . . . . . . . . . . . . . . . 7

3.6 Cryptic 3' Splice Sites _. . . . . . . . . . . . . . . . . . . . . . . . 8

3.7 Neighboring 5 Splice Sites . . . . . . . . . . . . . . . . . . . . 9

4 Algorithm Selection $\quad 10$

4.1 Background on Genetic Algorithms . . . . . . . . . . . . . . . . . . . 12

4.2 Selection Strategies . . . . . . . . . . . . . . . . . 15

4.3 Crossover operators . . . . . . . . . . . . . . . 16

4.4 Mutation . . . . . . . . . . . . . . . . . . 17

4.5 Replacement Strategies . . . . . . . . . . . . . . . . . . . . 17

5 Modeling the problem definition using GA 21

5.1 Data Model . . . . . . . . . . . . . . . . . . 21

5.2 Algorithms: GA . . . . . . . . . . . . . . . . 21

5.2.1 Fitness Function: Position Weight Matrix . . . . . . . . . . 22

5.2 .2 Search space formalisms . . . . . . . . . . . . . . . 24

5.2.3 Computing exact match ratio (EMR) . . . . . . . . . . 26

5.2 .4 Competing datasets . . . . . . . . . . . . . . . . . 26

5.2.5 Comparison Scores . . . . . . . . . . . . . . . . 27

5.2 .6 Enhanced Scores . . . . . . . . . . . . . . . . . . . . . . 27 


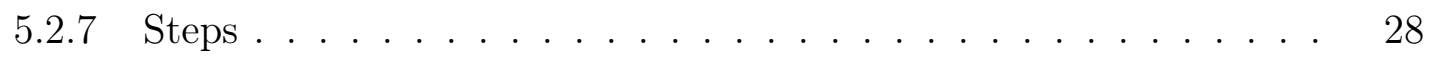

5.2 .8 Dataset Limitations . . . . . . . . . . . . . . . . . . . 28

5.3 Algorithms: Operator and Strategies . . . . . . . . . . . . . . . . 29

5.3.1 Selection Strategies . . . . . . . . . . . . . . . . 29

5.3.2 Crossover Operators . . . . . . . . . . . . . . . . 33

6 Results 37

6.1 Scoring Tables . . . . . . . . . . . . . . . . . . . . . . . 38

6.2 Scoring Tables: 5 . . . . . . . . . . . . . . . . . 39

6.3 Scoring Tables: 3' . . . . . . . . . . . . . . . . . . . . . . 42

6.4 Score Summaries . . . . . . . . . . . . . . . . . . . . . . 44

6.4.1 Point of Interest: CSCORE for Cryptic Splice Sites . . . . . . . . 45

$\begin{array}{lll}7 & \text { Analysis } & 47\end{array}$

$7.1 \quad 5$ Splice Sites . . . . . . . . . . . . . . . . . . . 47

7.2 3' Splice Sites . . . . . . . . . . . . . . . . . . . . 47

8 Phase 2: Classification Test Using Extrapolated Sets 48

8.1 Classification Primer . . . . . . . . . . . . . . . . . . . . 48

8.2 Random Forest Classifiers . . . . . . . . . . . . . . . . . . 50

8.3 Modeling the Random Forest Classifier . . . . . . . . . . . . . . . 51

9 Conclusion $\quad 54$ 


\section{List of Tables}

1 Input sequence lines from file EI_true.seq . . . . . . . . . 5

$2 \quad 9$-mers extracted from the sequences in Table $1 \ldots \ldots \ldots$

3 Input sequence lines from IE_true.seq . . . . . . . . . . . 8

$4 \quad 13$-mers extracted from the sequences in Table $3 \ldots \ldots$. . . . 8

5 Sample data: Roulette Wheel Selection . . . . . . . . . . . . . . . 29

6 Sample data: Ranked Selection _. . . . . . . . . . . . . . . . . 31

7 SELECTION: ROULETTEWHEEL; CROSSOVER: 2P: AUTH - CSCORE 39

8 SELECTION: ROULETTEWHEEL; CROSSOVER: UNIFORMORDERBASED: CSS - FITNESS . . . . . . . . . . . . . . . . 40 40

9 SELECTION: ROULETTEWHEEL; CROSSOVER: UNIFORMORDERBASED: NEIGHBOR - CSCORE . . . . . . . . . . . . . . . . 41

10 SELECTION: ROULETTEWHEEL; CROSSOVER: UNIFORMORDERBASED: AUTH - FITNESS . . . . . . . . . . . . . . . . . 42

11 SELECTION: TOURNAMENT; CROSSOVER: 1P: CSS - CSCORE . . 43

12 SUMMARY: 5' splice sites | pivot: dataname, metric . . . . . . . . . 44

13 SUMMARY: 3' splice sites | pivot: dataname, metric . . . . . . . . 45

14 SUMMARY: 5' - BEST GA PARAMETERS FOR CSS CSCORES . . . 46

15 SUMMARY: 3' - BEST GA PARAMETERS FOR CSS CSCORES . . . 46 


\section{List of Figures}

1 Protein synthesis . . . . . . . . . . . . . . . . . . 1

$2 \quad 5$ 'Splice Site . . . . . . . . . . . . . . . . . . . . . 3

3 HS3D downloads page ....................... 4

$4 \quad 3$ 'Splice Site . . . . . . . . . . . . . . . . . . . . 7

$5 \quad$ GA: general steps . . . . . . . . . . . . . . . 11

6 GA: search space . . . . . . . . . . . . . . . 11

$7 \quad$ Population and Individual dimensions . . . . . . . . . . . . . . . . . 12

8 Chromosome, Gene, and Alleles . . . . . . . . . . . . . 13

$9 \quad$ An algorithmic framework for genetic algorithms . . . . . . . . . . . . . 14

10 Simple GA with one-point crossover, roulette wheel selection, and delete all strategy . . . . . . . . . . . . . . . . . . 20

11 Position Weight Matrix: block diagram . . . . . . . . . . . . . 23

12 Roulette wheel for chromosomes in Table $5 \ldots \ldots$. . . . . . . . . 29

13 Example: One Point Crossover . . . . . . . . . . . . . . . . 33

14 Example: Two Point Crossover . . . . . . . . . . . . . . . . . . . . . 34

15 Example: Uniform Crossover . . . . . . . . . . . . . . . . 35

16 Example: Uniform Order-Based Crossover . . . . . . . . . . . . . . 36

17 Random Forest Classifier workflow for extrapolated splice sites . . . . . . 53 


\section{Motivation}

The eukaryotic genome consists of many genes. According to the latest results of the Human Genome Project, humans have approximately 20,000 protein-coding genes [1]. Transcription and translation are some of the important processes in the gene expression pathways. Transcription involves generation of messenger RNA (mRNA) from premessenger RNA (pre-mRNA). Both mRNA and pre-mRNA are sequences of nucleotides. The pre-mRNA is divided into consecutive nucleotide regions called introns and exons. An exon-intron boundary is called a 5' splice site and an intron-exon boundary is called a 3' splice spite. The introns are discarded and the exons are stitched together to form the mRNA.

The exact sequence of amino acids, generated by translation of the mRNA, dictates the structural properties of the protein molecule. The structure and composition of a protein molecule influence the physical and chemical properties exhibited by the protein. This process of synthesizing proteins is shown in figure 1 .

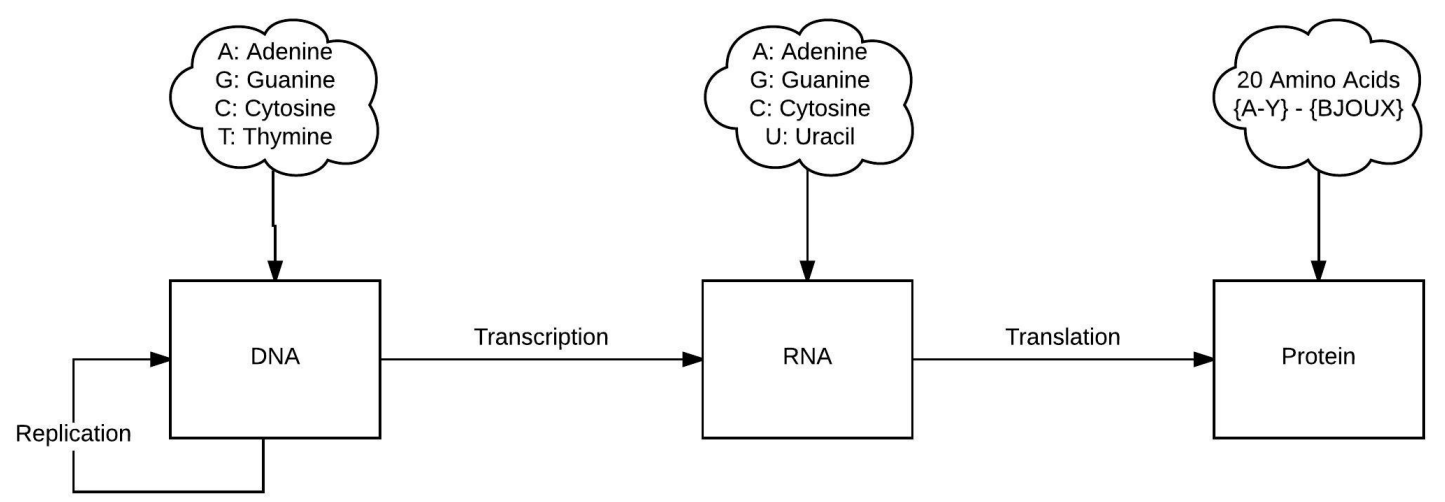

Figure 1: Protein synthesis

The proteins are responsible for various biological functions and traits of a eukaryotic organism. The splicing of pre-mRNA at 5' and 3' splice sites must be accurate in order to form the protein molecules that are required for satisfying the normally known functions of a healthy organism.

Mutations in the DNA can lead to one of many possible disruptions in the selection of 5' or 
3' splice sites. An obviously devastating disruption is a frame shift while extracting codons from the mRNA to form amino acid molecules. This may lead to a severely deficient expression of an important protein due to early detection of stop codons. In many cases, this leads to undesired consequences like fatal diseases. As per recent estimates, it is known that up to $50 \%$ of disease-causing mutations disrupt splicing.

The splice sites that are selected due to mis-splicing are called cryptic splice sites. However, there are multiple nucleotide subsequences in the pre-mRNA that contain candidate splice sites. Consequently, it is of crucial importance to understand the reasons behind the cryptic splice site selection by the spliceosome.

\section{Problem Statement}

To study the selection of splice sites by the spliceosome, three data sets were built, consisting of authentic, cryptic, and neighboring 5' splice sites. The data sets contain thousands of 9-mers: sequences that are 9 bases long. The natural splice sites are called authentic splice sites. Cryptic splice sites are activated when the natural splice sites are disrupted due to mutations. The authentic and cryptic splice sites are extracted from public datasets. The neighboring splice sites are extracted from within 100 base-pairs upstream and downstream of each cryptic splice site.

The primary goal is to build probabilistic models from each data set and quantitatively compare their similarities and differences. The primary hypothesis is that the authentic and cryptic splice sites are inherently different. The secondary hypothesis is that the neighboring splice sites are dissimilar to both authentic and cryptic splice sites. If probabilistic models are a good fit for imitating the behavior of the spliceosome, we can understand why the spliceosome chooses cryptic splice sites and discards neighboring splice sites when mutations disrupt the authentic splice site. 


\section{Data Specifications}

The introns and exons are consecutive alternating regions along the RNA separated by splice sites. The splice sites occurring along the exon-intron boundaries are called 5' splice sites. The splice sites occurring along the intron-intron boundaries are called 3' splice sites. For each of 5' and 3' splice sites, the natural splicing locations are called authentic splice sites. Cryptic splice sites are activated when mutations disrupt the authentic splice sites.

\subsection{5' Splice Sites}

The 5' splice sites are extracted based on the location of the invariant GT di-nucleotide. The authentic splice sites are extracted from the Homo Sapiens Splice Sites database (HS3D) $[2,3]$. The cryptic splice sites and neighboring splice sites are extracted from the DBASS5 database $[4,7]$.

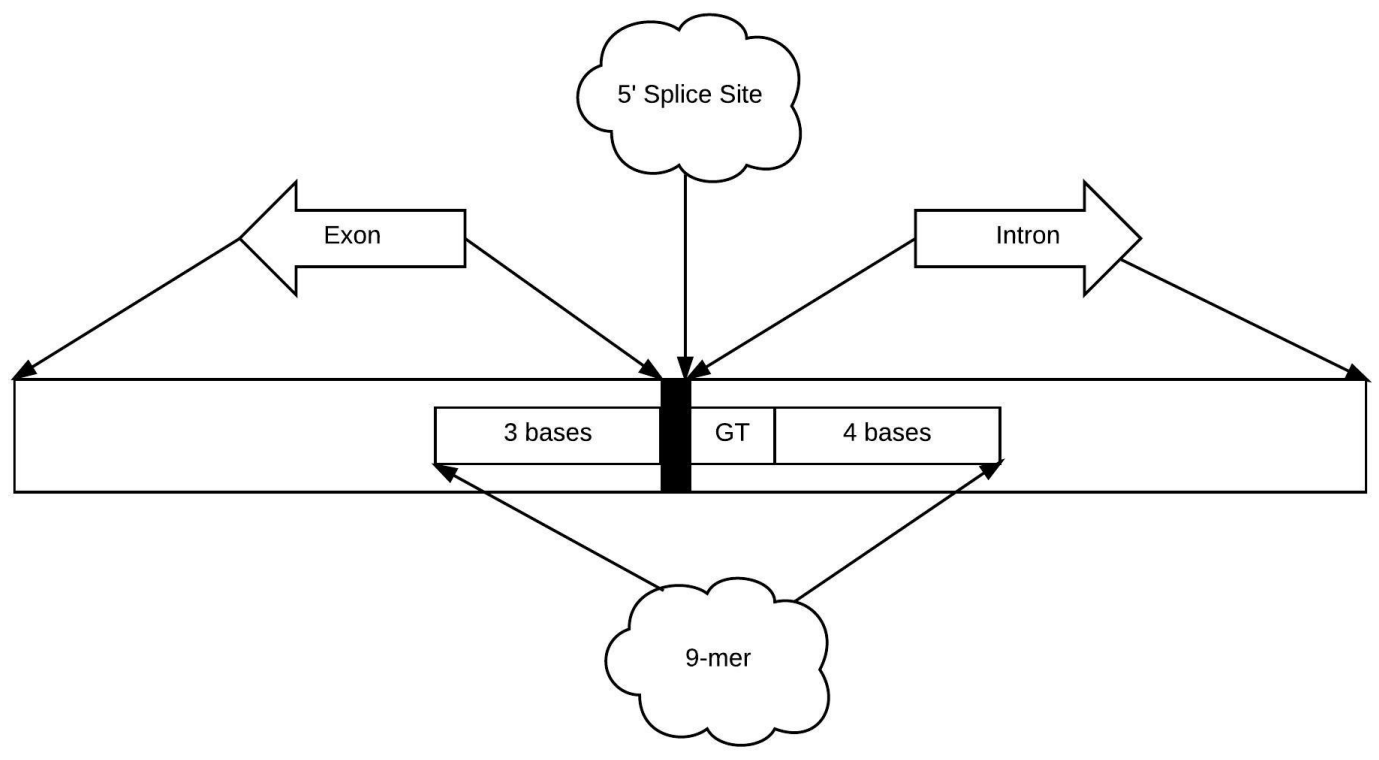

Figure 2: 5' Splice Site

\subsection{Authentic 5' Splice Sites}

HS3D offers the following files listed in figure 3 for download: 


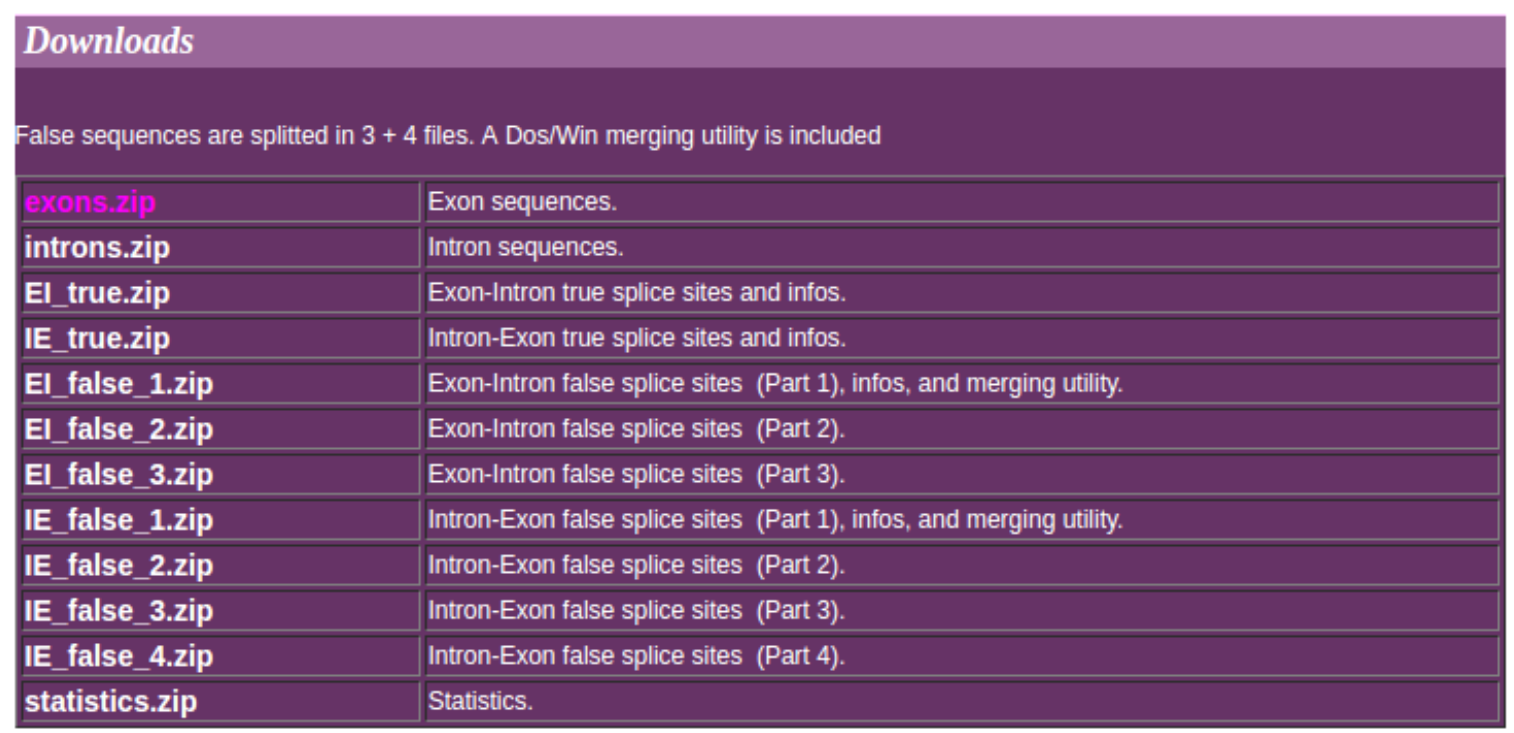

Figure 3: HS3D downloads page

Since 5' splice sites occur at the exon-intron boundary, the file EI_true.zip is relevant for Authentic 5' splice sites. The data is provided as a 'seq' file with 140 nucleotides in each line. Manually inspecting all the sequences reveals that the 5' consensus GT dinucleotide that marks the start of the intron is at position 71-72 in each sequence. The desired 5' splice site 9-mer comprises the last 3 nucleotides from the exon, the GT dinucleotide from the start of intron and the 4 nucleotides following it.

Table 1 shows a sample of 9-mers extracted from the dataset: 
Table 1: Input sequence lines from file EI_true.seq

AB000381 ( $1,2,2):$ CTCCTCTTTGCCTTACTCCTAGCCATGGAGCTC
CCATTGGTGGCAGCCAGTGCCACCATGCGCGCTCAGTGTAAGT
ATCATTCCCTCTCACTGTCCTGGAGAGGACGAGAATTCCACCT
GGGGTGCTGGGGGTCACTGGG

AB000381 (2,3,3): AATGACTTCAACTGTCCCAACATTAGAGTATGT CCGTATCATATTAGGCGCTGTATGACAATCTCCATTCGTAAGT ACCTCTTGGTCATTTGGACACATTGTAGATTAGTCCCCTACCT GGGTAGTTTCTGGGGCCAGGG

AB002059 (3,1,1): TGACCAGGAACTGGCGGGTGGGCGCCCTGCAGA GGCTGCTGCAGTTTGGGATCGTGGTCTATGTGGTAGGGTAAGA GAGAAGAGCTTTTGGCCAGGCTGGAGGGGCAAGGGAAGAGGTG GGGGGTGGGGCTTGGTCCTGC

AB002059 ( 4,2,2): TTCCGTCACTCAGATCAAGGAGCTTGGAAACCG GCTGTGGGATGTGGCCGACTTCGTGAAGCCACCTCAGGTGGGG GCCCTGATGTTGCTGACGGGGGCGCAAGTCCTTTCCCCACTGA CAGCCTGAACACCCGCCATGC

Table 2: 9-mers extracted from the sequences in Table 1

\begin{tabular}{|l|}
\hline AGTGTAAGT \\
\hline TTCGTAAGT \\
\hline AGGGTAAGA \\
\hline CAGGTGGGG \\
\hline
\end{tabular}

The total number of authentic 5' splice site 9-mers extracted from HS3D are $\mathbf{2 7 9 6 .}$

\subsection{Cryptic 5' Splice Sites}

The cryptic 5' splice sites are collected from the DBASS5 database portal [4,7] by crawling all available splice site details pages. These pages contain the nucleotide sequence and 
the context of the mutation that alters the splicing location. The mutation is indicated in the sequence with a greater than symbol.

For example: $(\mathrm{G}>\mathrm{A})$ means $\mathrm{G}$ is replaced by $\mathrm{A}$

The cryptic splice sites are denoted by the slash symbol.

Consider the following nucleotide sequence extracted from the first record.

URL: http://www.dbass.org.uk/DBASS5/viewsplicesite.aspx?id=627 (last retrieved: 04/23/2017)

CCAGCAACTT GGCTCTTTTT GGAGAGCGGC TGGGCCTGGT TGGCCACAGC CCCAGTTCTG CCAGCCTGAA CTTCCTCCAT GCCCTGGAGG TCATGTTCAA ATCCACCGTC CAGCTCATGT TCATGCCCAG GAGCCTGTCT CGCTGGACCA GCCCCAAG/G TGTGGAAGGA GCACTTTGAG GCCTGGGACT GCATCTTCCA GTAC $(\mathbf{G}>\mathbf{A})$ g tgaggccagg gaccogggca gtgctatggg gaagggacac catgggggcc caatttctcc ctctccacca cccagtgggg aatggaggcc acagggaggg gtcggggatt cctcaccttc ctgccaggga gattggtgcg aggctgggge tgggctggge tgatccggag aatttgggat gagagcaggg agacttgggt gtcggggcag tctgggcagg aggaggacac tgaaggatgt ctccagcac caaagtctga gggctgcctc ccgctccccg

In the above nucleotide sequence:

- Capital nucleotide symbols indicate the trailing part of the exon

- Small nucleotide symbols indicate the leading part of the intron

- Mutation is marked as : $(\mathrm{G}>\mathrm{A})$

- Cryptic splice site is marked as : "GCCCCAAG/G TGTGGAAGG"

The cryptic 5' splice site 9-mer extracted from the above sequence is: AAGGTGTGG The total number of cryptic 5' splice site 9-mers extracted from DBass5 are $\mathbf{5 3 9 .}$

\subsection{3' Splice Sites}

The 3' splice sites are extracted from the HS3D and DBASS3 databases based on the location of the invariant AG dinucleotide. There is an important $Y_{n}$ consensus that 
precedes the AG dinucleotide. $\mathrm{Y}$ is the notation for a pyrimidine and $\mathrm{n}$ is the length of the consensus sequence and its value for 3' splice sites is approximately 10 . The authentic 3' splice sites are extracted from the Homo Sapiens Splice Sites database (HS3D) [2,3]. The cryptic 3' splice sites and neighboring splice sites are extracted from the DBASS3 database [4-6].

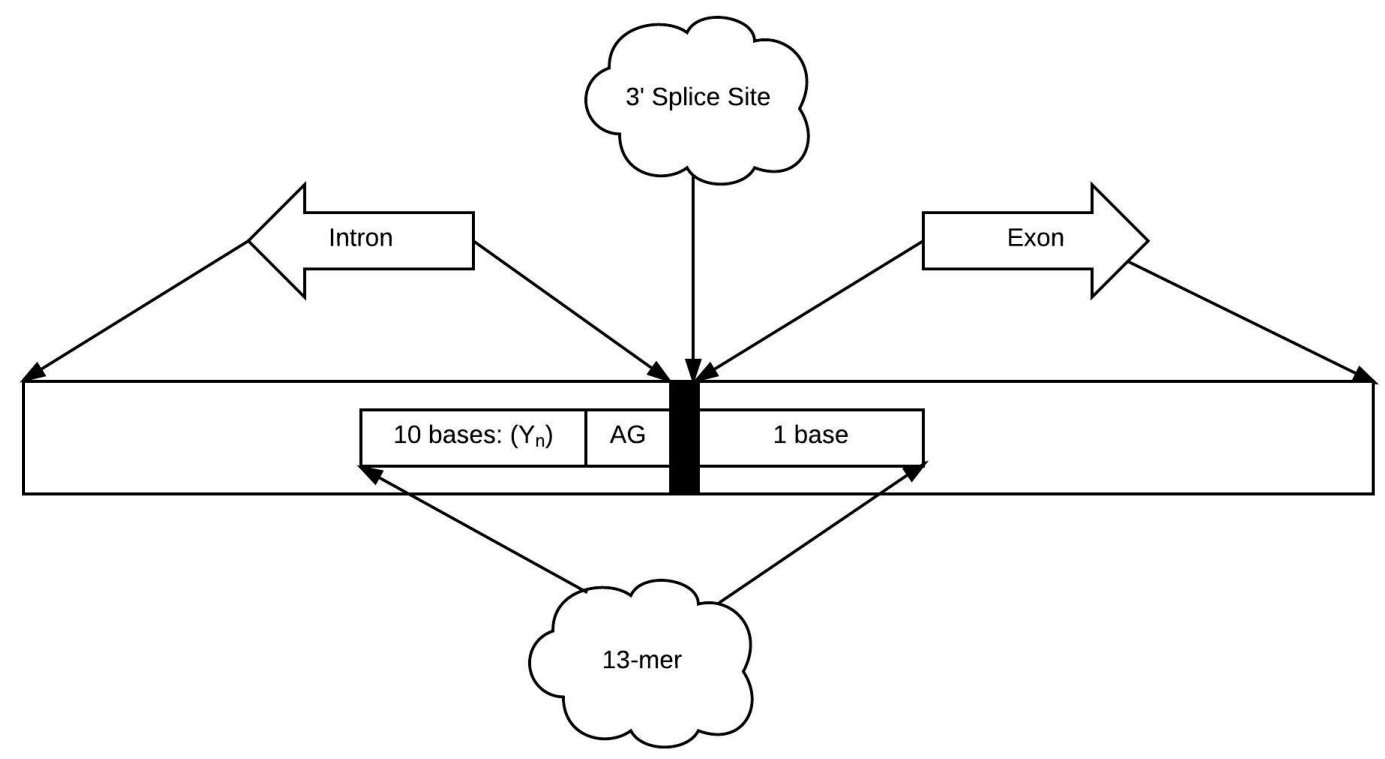

Figure 4: 3' Splice Site

\subsection{Authentic 3' Splice Sites}

Since 3' splice sites occur at the intron-exon boundary, the file IE_true.zip is relevant for Authentic 3' splice sites. This data is also provided as a 'seq' file with 140 nucleotides in each line. Manually inspecting all the sequences reveals that the 3' consensus AG dinucleotide that marks the end of the exon is at position 69-70 in each sequence. The desired 3' splice site 13-mer comprises of the last 12 nucleotides from positions 59-70 at the end of the exon, and one nucleotide from the start of the intron.

Table 3 shows a sample of 13-mers extracted from the dataset: 
Table 3: Input sequence lines from IE_true.seq

AB000381 $(1,1,2)$ : GGCCAGGGGCATAGAGCTGGCCAAGGAGCCATGGCTCAC

TAACGTGTTGTATGGGGCTCCTTCCCTTCAGGTCCAGGCTCCTGCGTGAAG TGATGCTCCTCTTTGCCTTACTCCTAGCCATGGAGCTCCCATTGGTGGCA AB000381(2,2,3): GAGTGAGCTGGTAATGGGTGGAAAAGGCGTAGTGGAGCA GAAGCCTGAAGCCTGCTTTCTCCCCTCTCAGGGACTTACAGTTTGAGATGC CATGACTGTGCGGTCATAAATGACTTCAACTGTCCCAACATTAGAGTATG AB000381(3,3,4): TGTATGTGCCTCAATATTTACAAGCAGAAAATGTGAAAT CAATTATTTTCATTGCTGCTTCTTTTTтTAGGCATAAATTCTCGTGAACTAC TTGTTTATAAGAACTGTACAAACAACTGCACATTTGTATATGCAGCTGA AB002059(4,1,2): GTAGGGCTCAGCTCCGCCCCTGTCACTACACGCTGGGGA CACACCACACTGCCCGACTTCTCCTCCCCAGGTGGGCGCTCCTCGCCAAAAA AGGCTACCAGGAGCGGGACCTGGAACCCCAGTTTTCCATCATCACCAAA AB002059(5,2,3): AGGCTGCCGGCTTCCGGCCTTTCCAGTCAACACGAGCCC AGCCAGGCCAACCTTGAGACTTGCCTCCTAGGGAGAGAACGTGTTCTTCTTG GTGACCAACTTCCTTGTGACGCCAGCCCAAGTTCAGGGCAGATGCCCAG

Table 4: 13-mers extracted from the sequences in Table 3

\begin{tabular}{|l|}
\hline CCTTCCСTTCAGG \\
\hline CTCCСCTCTCAGG \\
\hline TTCTTTTTTTAGG \\
\hline TCTCCTCCCCAGG \\
\hline CTTGCCTCCTAGG \\
\hline
\end{tabular}

The total number of authentic 3' splice site 13-mers extracted from HS3D are $\mathbf{2 8 8 0 .}$

\subsection{Cryptic 3' Splice Sites}

The cryptic 3' splice sites are collected from the DBASS3 database portal [4-6] by crawling all available splice site details page. The fields on the DBASS3 splice site details pages 
are similar to the ones on the DBASS5 pages.

The desired 13-mer comprises of :

- 10 nucleotides before the ag dinucleotide from the intron

- the ag dinucleotide at the splice site marker from the intron

- one nucleotide after the splice site marker from the exon

Consider the following nucleotide sequence extracted from a record at URL: http://www.dbass.org.uk/DBASS3/viewsplicesite.aspx?id=52 (last retrieved: 04/23/2017)

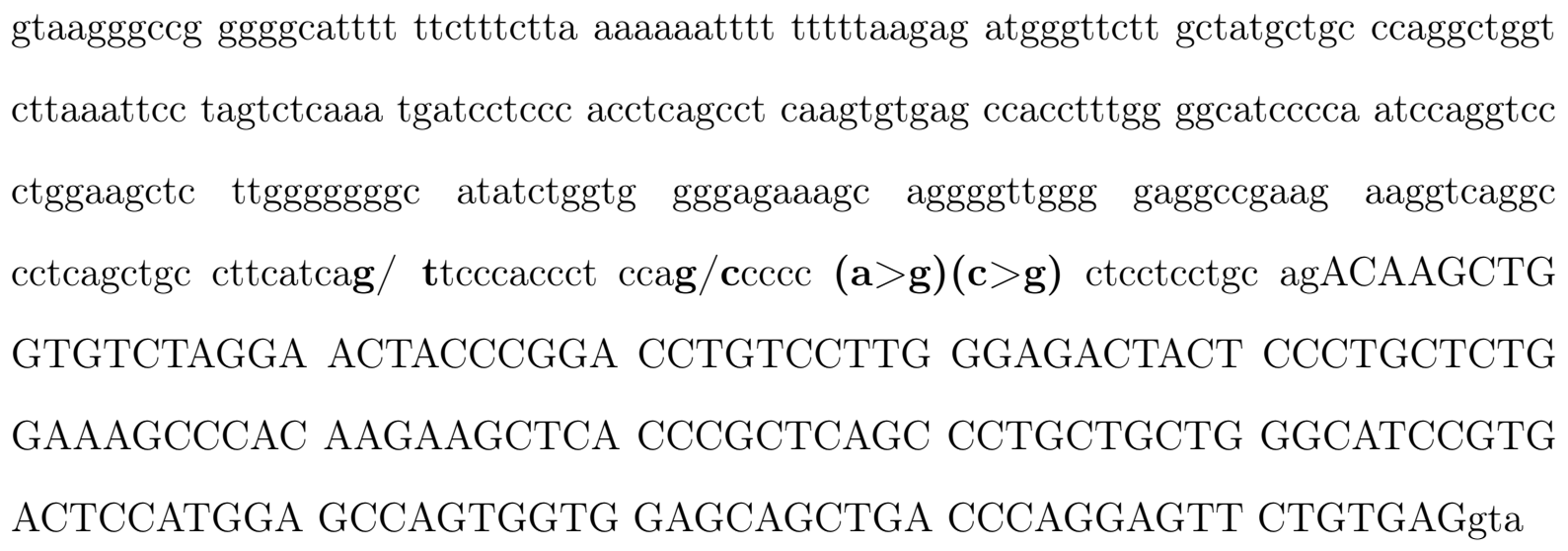

In the above nucleotide sequence:

- Capital nucleotide symbols indicate the leading part of the exon

- Small nucleotide symbols indicate the trailing part of the intron

- Mutations are marked as : (a $>\mathrm{g})$ and $(\mathrm{c}>\mathrm{g})$ Cryptic splice sites are marked as : "cagctgc cttcatcag/ ttcccacct ccag/cccc"

There are two cryptic 3' splice site 9-mers extracted from the above sequence. They are: "tgccttcatcagt" and "cccaccctccagc"

The total number of cryptic 3' splice site 13-mers extracted from DBASS3 are $\mathbf{3 0 6}$.

\subsection{Neighboring 5' Splice Sites}

The putative splice sites around the known cryptic splice sites are called neighboring splice sites. These are the putative splice sites that were not selected for splicing by 
the spliceosome in the event of a mutation. Using the nucleotide sequences extracted from the DBASS5 splice site details pages. The 100 base-pairs upstream and 100 basepairs downstream of the splice site are parsed to look for the GT dinucleotide. All such occurrences are captured in the neighboring 5' splice site dataset in the form of 9-mers with the GT dinucleotide as the 4th and 5th bases.

The total number of neighboring 5' splice site 9-mers extracted from DBASS5 are 2213. The next section emphasizes the importance of analyzing the search space defined by these datasets. A grass-root approach is used to select a suitable algorithm for achieving the objectives set in the problem definition.

\section{Algorithm Selection}

For the 5' and 3' splice sites, we can model the problem statement as a search problem. The spliceosome binds to a specific subset of the entire 9-mer search space. The cardinality of each position in the 9-mer is four (equivalent to A, C, G, and T). In 5' splice sites, positions 4 and 5 are always occupied by the GT dinucleotide. Hence, the size of the $5^{\prime}$ splice site search space is $4^{7}=16384$. This search space includes the authentic, cryptic, and neighboring splice sites.

Evolutionary Algorithms (EAs) are applicable to problems that have no known classical optimization methods [8]. If a traditional method is applicable to solve a problem, then EA should not be used since traditional methods are more efficient in such a case. Since we do not understand the choices of a spliceosome completely, EAs are suitable for this problem. Section 5 describes a Genetic Algorithm based approach in more detail. 


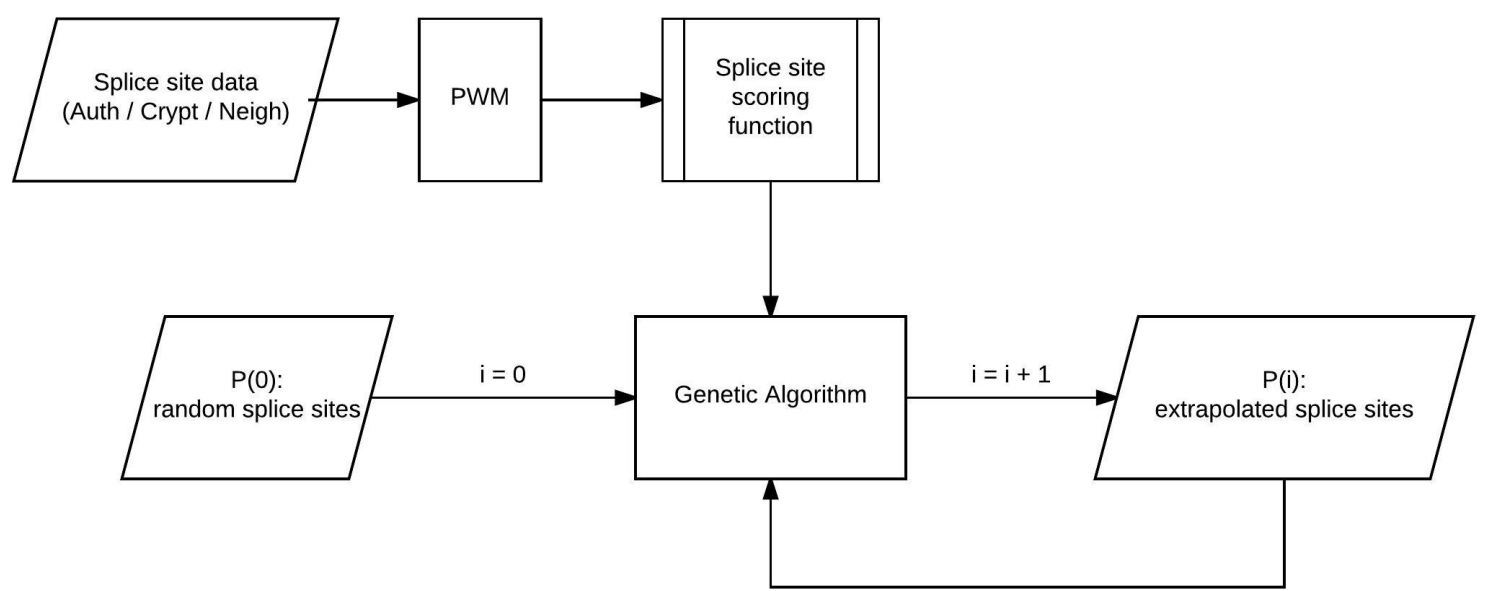

Figure 5: GA: general steps

Figure 5 shows a general iterative approach that is used to explore the splice site search space in a guided manner.

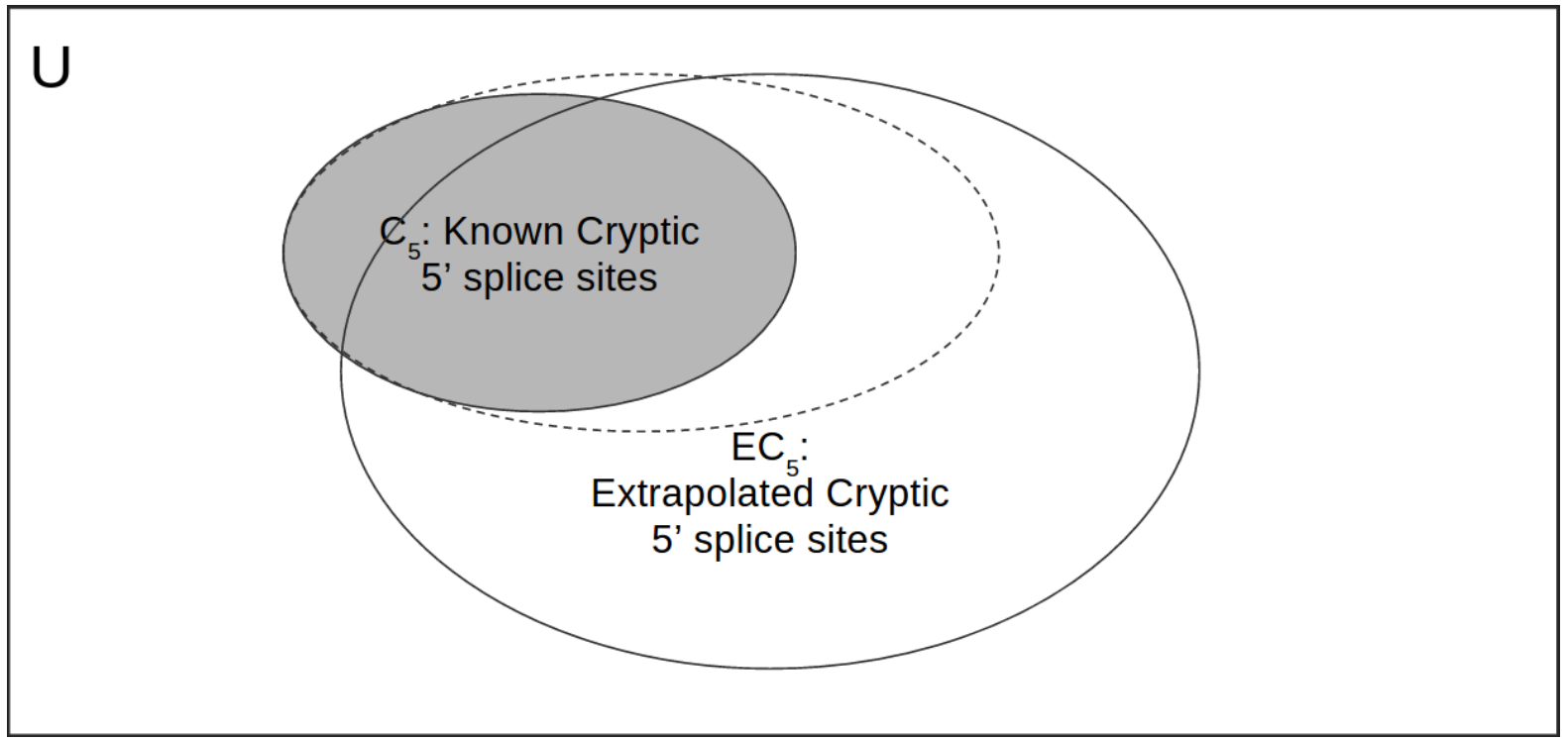

Figure 6: GA: search space

Figure 6 depicts the search space of all cryptic 5' splice sites. The entire search space is denoted as U. The known cryptic splice sites are represented by the shaded region. The dotted region represents all undiscovered actual cryptic 5' splice sites. Multiple iterations of a genetic algorithm framework are performed to extrapolate putative 5' splice sites that have a high probability of also being cryptic. This is represented by the outer larger region 
labeled $E C_{5}$.

\subsection{Background on Genetic Algorithms}

Genetic Algorithms were first proposed and analyzed by John Holland [10]. Genetic Algorithms are a type of EA that deal with search and optimization based on a fitness function. They follow the philosophy of survival of the fittest by imitating the mechanisms of natural biological selection and genetics.

Genetic algorithms follow a generic domain-agnostic framework. Hence, it has the advantage of being applicable to many problems. Some of the features that initially separated GA from other evolutionary approaches are:

- Bitstring representation

- Proportional selection

- Crossover

Although the representation and selection methods have advanced significantly, there is still a large emphasis on the crossover operation. Crossover is known to give GAs a distinctive advantage over other methods [8].

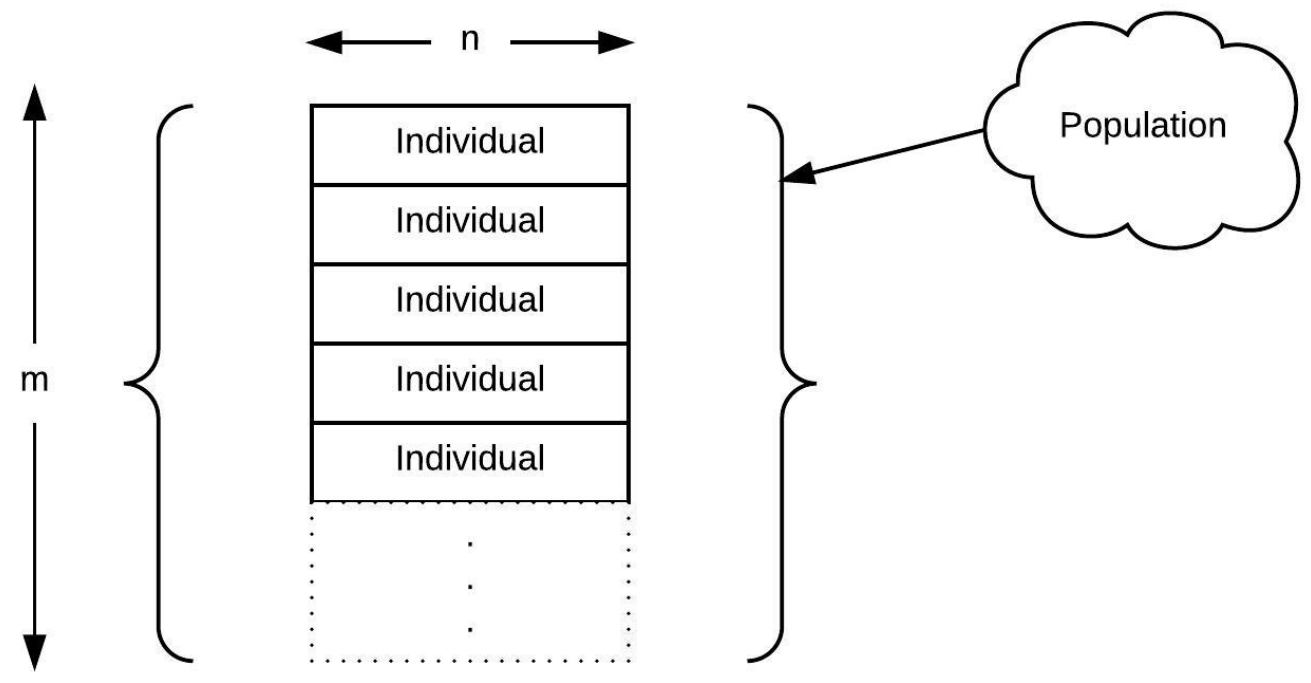

Figure 7: Population and Individual dimensions 


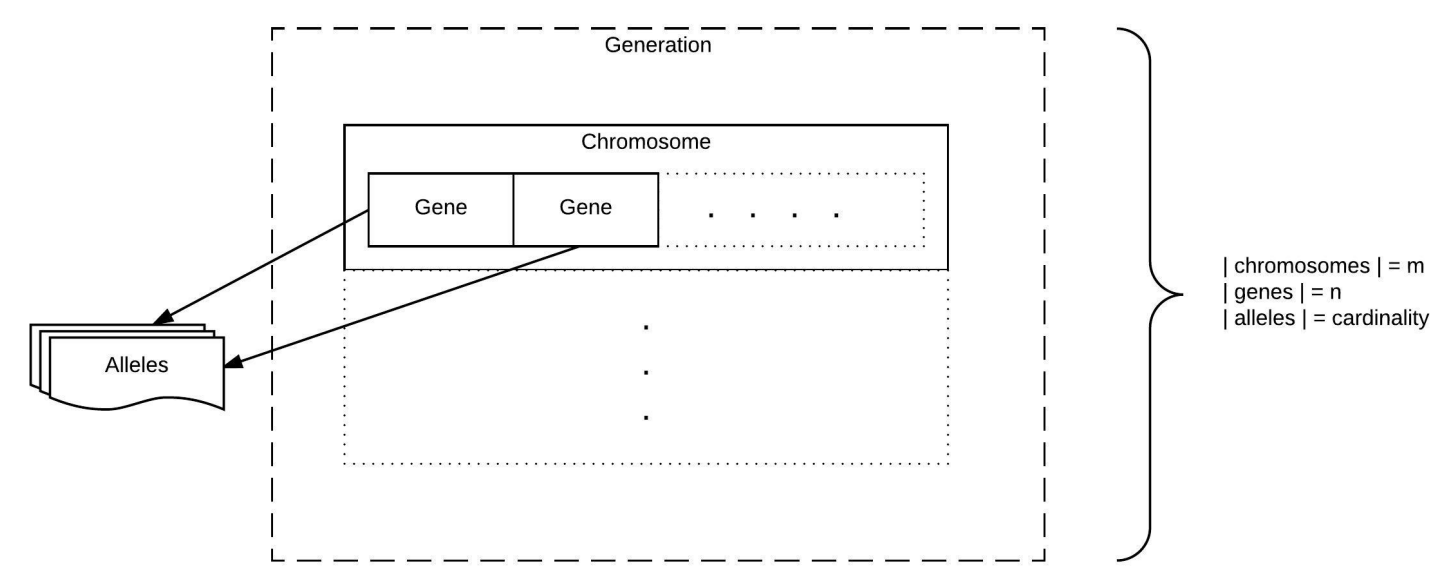

Figure 8: Chromosome, Gene, and Alleles

Basic terminology (Refer figures 7 and 8):

- Generation: A population of fixed size $m$

- Chromosome: An individual of length $\mathrm{n}$ in the population

- Gene: Each of the $\mathrm{n}$ positions in a chromosome is called a gene. It can be treated as a variable

- Allele: A value from a fixed set that can be assigned to a gene 


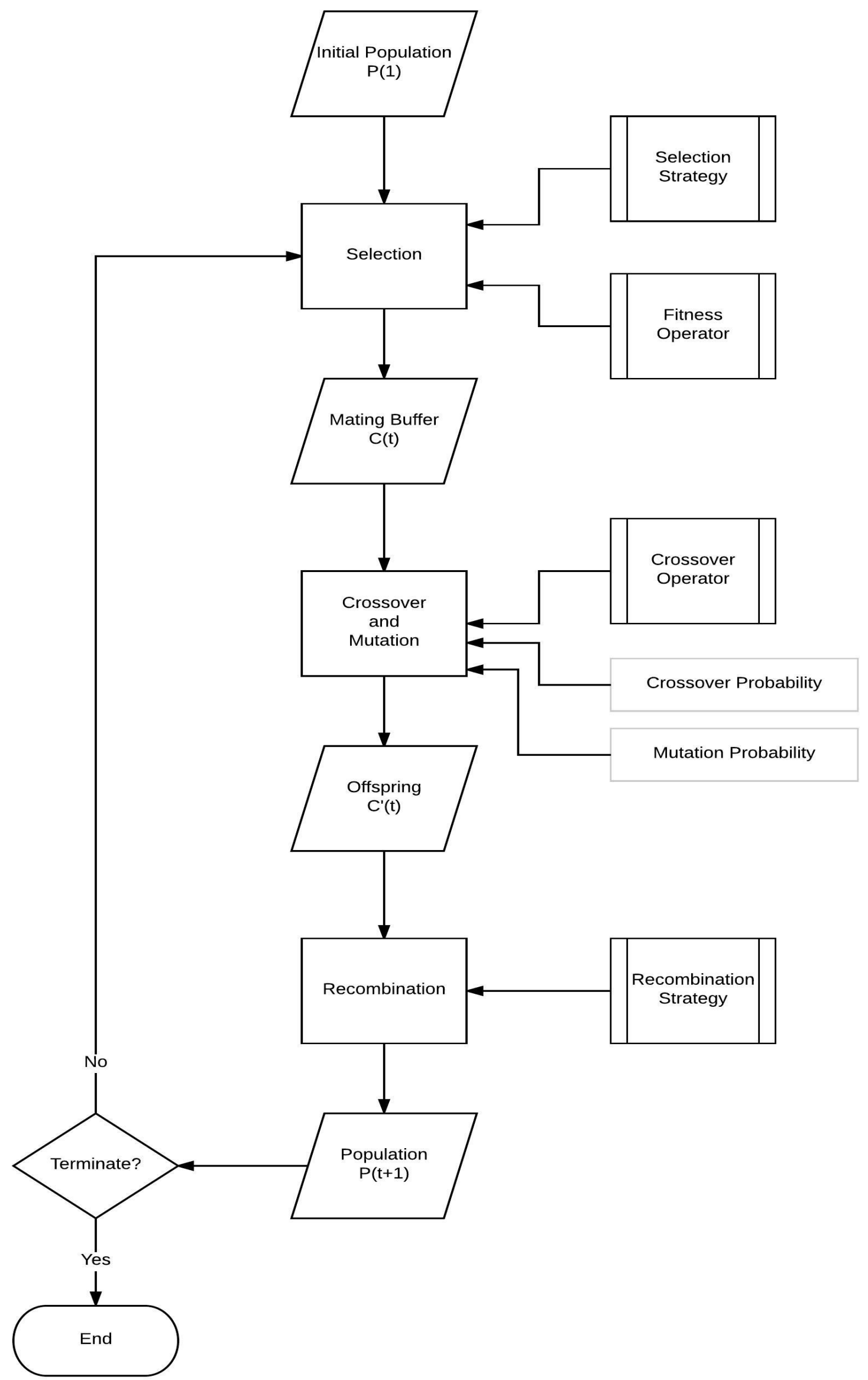

Figure 9: An algorithmic framework for genetic algorithms 
Genetic Algorithms are applicable to many problems because they make very few domainspecific assumptions. Figure 9 shows a generic algorithmic framework for domain-agnostic genetic algorithms. Each of the blocks in the figure can be briefly explained as follows:

- Fitness: It is responsible for quantifying the health of chromosomes. Good chromosomes are fitter chromosomes with higher fitness values

- Selection: It is responsible for stochastically retaining good chromosomes based on their fitness to form a mating population

- Crossover: It combines pieces of traits from good parent chromosomes to form offspring chromosomes

- Mutation: It randomly changes individual genes in the offspring chromosomes to perform random walks in the search space

- Recombination: It combines the offsprings with the parent population to create a new population

There are many strategies and operators that can be selected according to the target problem. The framework specified in figure 9 supports customization of the strategies and operators. It makes no assumptions about the initial population, chromosome representation, or the operators. Selection and recombination are the two phases that can be used to introduce bias [8].

\subsection{Selection Strategies}

Selection strategies are responsible for selecting individuals from input population to form a mating population. Selection strategies play an important role in the survival of chromosomes with good traits. Thus, it plays the role of selection pressure. Selection pressure is governed by the differences in selection probabilities of individual chromosomes. If the differences are too small then the selection pressure is weak.

1. Roulette wheel:

The total fitness value of all individuals in the parent population is computed. The 
selection probability of each individual is calculated as the ratio of its fitness to the total fitness. However, this strategy may lead to weak selection pressure.

The workaround for weak selection pressure is to scale the fitness probabilities with respect to the worst fitness. However, this may lead to excessive selection pressure. The best individual may take over the entire population within a few iterations [8].

2. Ranked selection:

The workaround for excessive selection pressure is to use ranked selection. The parent population is sorted by fitness. The probability of selection is a linear function of the ranks sorted by fitness.

3. Tournament selection:

A small subset of the parent population is selected at random and the individual with the best fitness is chosen for mating. This is repeated $m$ times. The selection pressure can be controlled by adjusting the set size.

4. Elitist strategy:

This is more of a strategy post selection. All but one of the child population after selection are chosen. The best individual from the parent population is added to the child population.

\subsection{Crossover operators}

Crossover operator combines features from two highly fit individual chromosomes to form offspring chromosomes. The individuals maybe highly fit due to different reasons. However, we do not know which features account for the high fitness. Hence, the features are combined at random [9].

Types of crossover [9]:

- One-point crossover

- Two-point crossover

- K-point crossover 
- Uniform crossover

- Uniform order-based crossover

- Order-based crossover

- Partially matched crossover (PMX)

- Cycle crossover (CX)

For more details on each type of crossover, refer to how they are used with 9-mer data in section 5.3.2.

\subsection{Mutation}

Mutation is the mechanism for producing variations by randomly replacing one allele with another. A commonly used rate of mutation is one over the length of the string. It leads to random walk traversal of the search space.

\subsection{Replacement Strategies}

A child population is generated from the parent population $\mathrm{P}(\mathrm{t})$ using selection, crossover, and mutation operations. A suitable replacement strategy is required to form the population for the next generation $\mathrm{P}(\mathrm{t}+1)$ using candidate individuals from both the parent and child populations [8].

Some of the notable GA implementations that implement complex replacement strategies are:

- Whitleys GENITOR

- Syswerda's steady-state GA

- Eshelman's CHC

- Mühlenbein's breeder GA 
Replacement Strategy Abstractions: [8]

Let us assume:

- $\mu$ : the set of chromosomes included in the parent population

- $\lambda$ : the set of chromosomes included in the child population

1. $(\mu+\lambda)$ Evolution Strategies (ES):

The $\mu$ parents and $\lambda$ children are merged and the best $\mu$ individuals are chosen to form the new parent population.

2. $(\mu, \lambda) \mathrm{ES}:$

The $\mu$ parents produce $\lambda$ offsprings such that $\lambda>\mu$. The best $\mu$ offsprings out of the $\lambda$ offsprings are chosen to form the new parent population.

3. Other Variations: Two other degrees of variation in a replacement strategy:

- Number of matings per iteration:

Whether the GA produces one or two versus many $(\mu)$ offsprings in each iteration; De Jong and Sarma (1993) claim that the main difference between variations in the number of allowed matings is that a strategy with fewer matings leads to higher variance in performance.

- Whether the replacement strategy is biased or not:

In case of an unbiased replacement strategy, if all the parents are replaced by the children, then we risk losing good individuals from the parent population for good. An advantage of this strategy is that the algorithm may wander out of a local minimum.

4. Practical Replacement Strategies [9]

- Delete All:

All the child population replaces the entire current population 
- Steady-state:

Only $n$ members of the current population are replaced by members from the child population; The quantity $n$ and the strategy for removal are parametrized

- Steady-state-no-duplicates:

Same as the Steady-state strategy except that the algorithm checks for duplicates while introducing chromosomes from the child population into the current population 


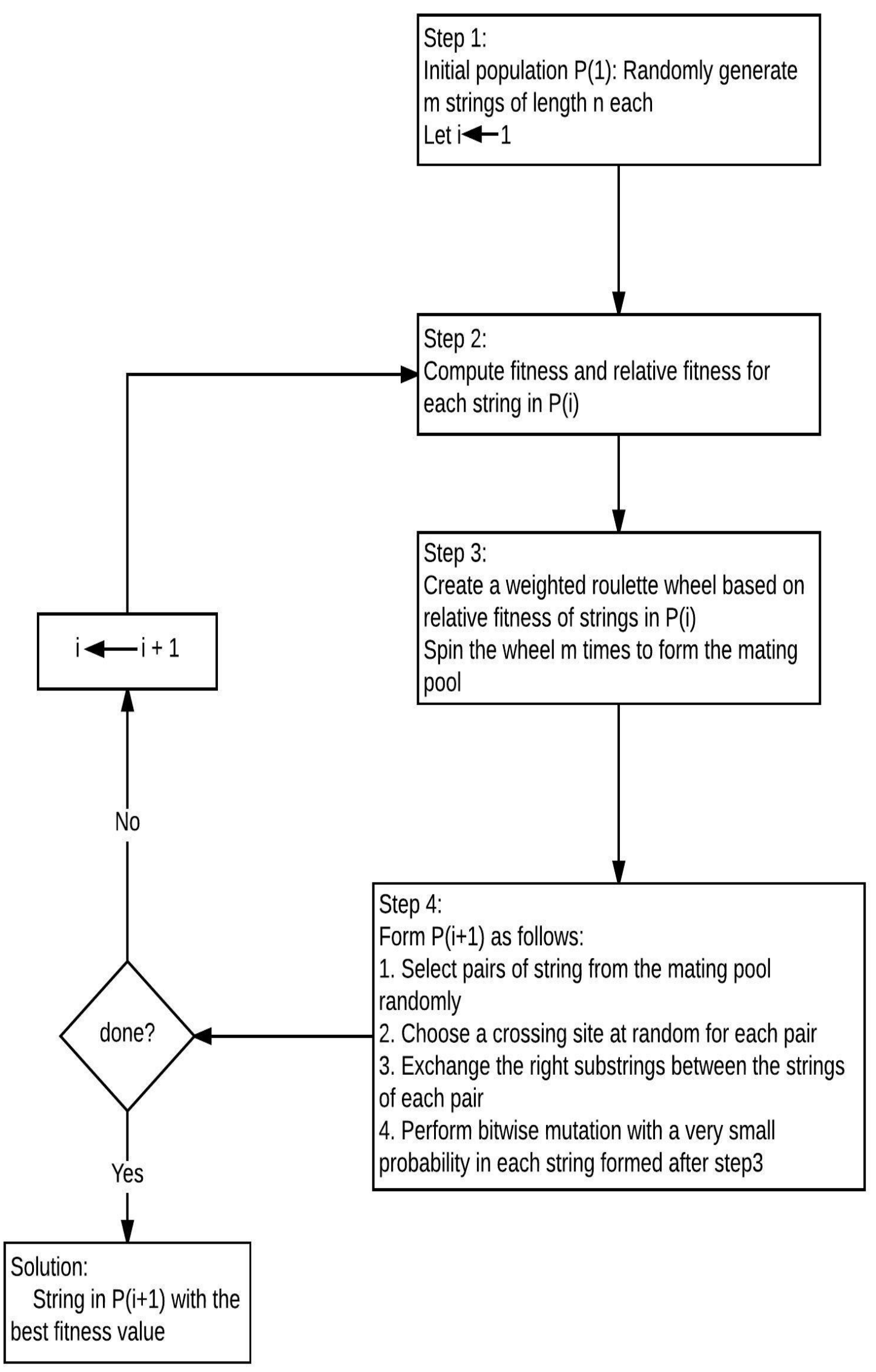

Figure 10: Simple GA with one-point crossover, roulette wheel selection, and delete all 
The selection strategies and crossover operators described in this section can be applied to the 5' and 3' splice site datasets, described in section 3, using the algorithms specified in the next section.

\section{Modeling the problem definition using GA}

\subsection{Data Model}

Following is a logical mapping of the terminology shown in figure 6 to the splice site n-mer data:

- chromosome: an entire n-mer

- gene: each base in the n-mer

- allele: each base in the n-mer can take one of the following four nucleotides (A: Adenine, C: Cytosine, G: Guanine, T: Thymine)

Following are the encoding formats for 5' and 3' splice sites:

- 5' splice site 9-mer A chromosome for a 5' splice site 9-mer is represented as a string of length 9 where each character belongs to the set $\{A, C, G, T\}$

E.g.: AAAGTGTCC

- 3' splice site 13-mer A chromosome for a 3' splice site 13-mer is represented as a string of length 13 where each character belongs to the set $\{A, C, G, T\}$

E.g.: AGATTCTCCCAGA

\subsection{Algorithms: GA}

The high-level generic flow of the algorithm is as follows:

1. Build a probabilistic model of the input dataset

2. Start with a random population 
3. Extrapolate a subset of the search space using the probabilistic model built in step 1

4. Determine how much of the input dataset is covered by the subset of the search space extrapolated in step 3

\subsubsection{Fitness Function: Position Weight Matrix}

Position Weight Matrix (PWM) is a popular bioinformatic tool for predicting motifs in a sequence of nucleotides. It has also been found useful in many other applications involving sequence patterns and local multi-alignments. PWM has been successfully applied to the representation of splice sites in nucleotide sequences [15]. The PWM score of a motif is a useful measure of its health [14]. 


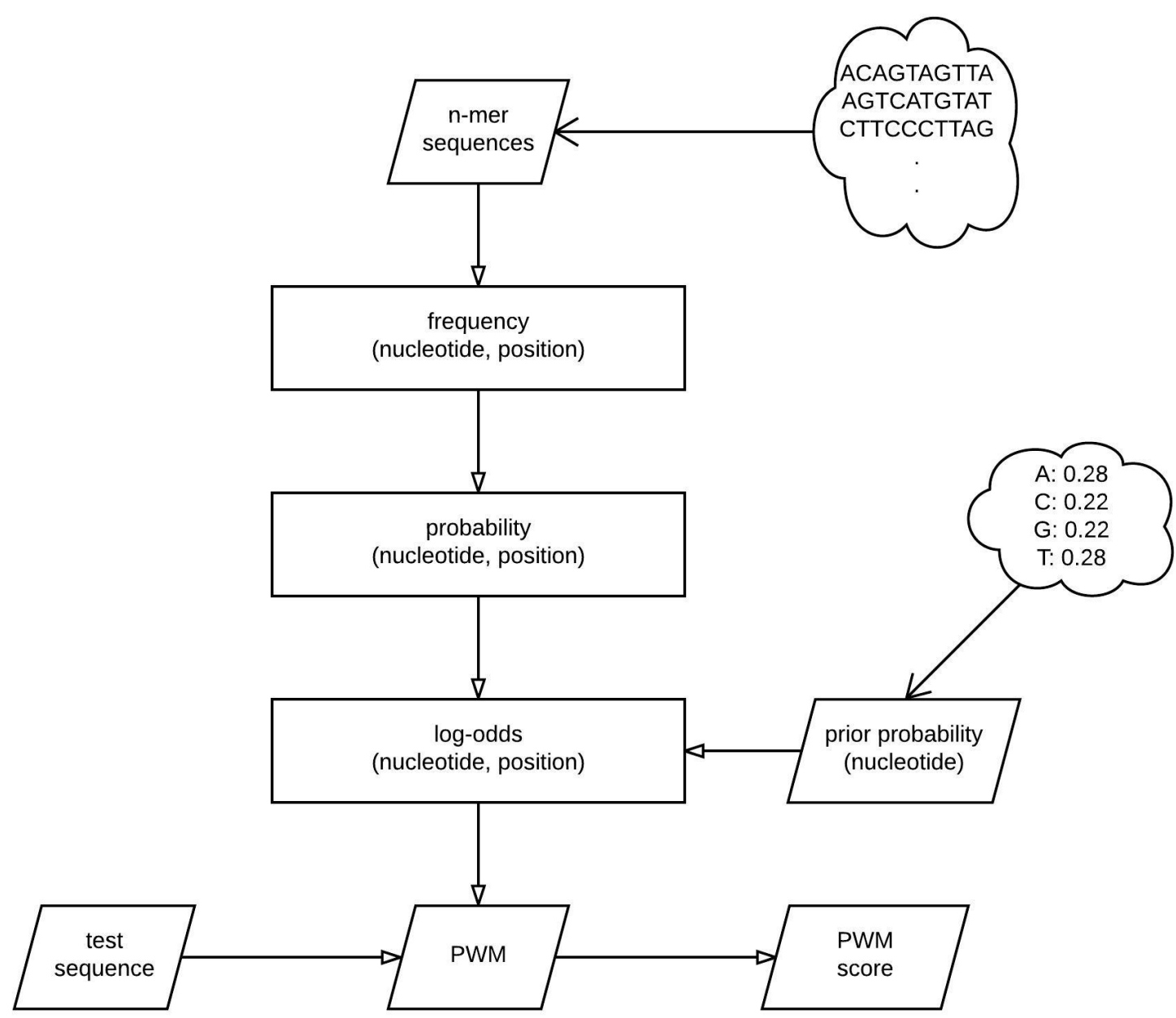

Figure 11: Position Weight Matrix: block diagram

figure 5.2.1 shows the different phases involved in computation of a PWM from nucleotide sequences.

\section{Algorithm: PWM}

Given Inputs:

- Nucleotide sequences

- Nucleotide prior probabilities

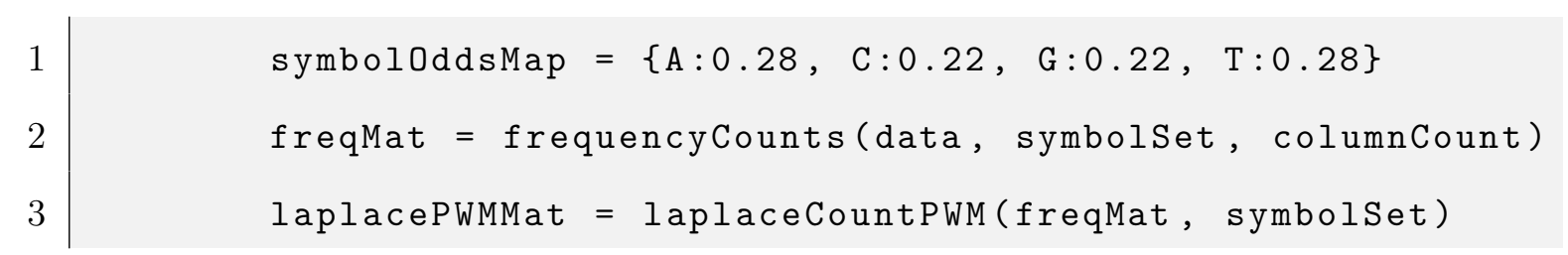




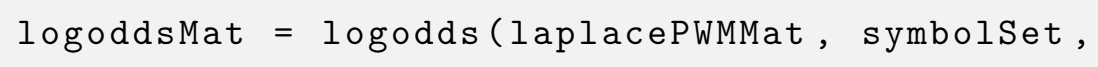

The log-odds matrix computed in step 4 is the PWM and can be used to score unknown sequences.

Laplace pseudo counts is used to handle zero values in step 3.

The log-odds matrix is the logarithm of a likelihood ratio. Hence,

score $>0 \Rightarrow$ positive sample

score $<0 \Rightarrow$ negative sample

\subsubsection{Search space formalisms}

The input dataset comprises of the following types of splice sites:

- $A_{5}$ : All known authentic 5' splice sites

- $C_{5}$ : All known cryptic 5' splice sites

- $N_{5}$ : All known neighboring 5' splice sites extracted as specified in section 3.7

- $A_{3}$ : All known authentic 3' splice sites

- $C_{3}$ : All known cryptic 3' splice sites

For the sake of simplicity, let us consider only the ${ }^{\prime}$ ' splice site datasets: $A_{5}, C_{5}$, and $N_{5}$.

Let $S_{5}$ be the entire search space of 5 ' splice sites.

$$
\left|S_{5}\right|=4^{7}=16384
$$

Without making any assumptions about the relation that exists between each dataset and $S_{5}$, we can state:

$$
\begin{aligned}
& A_{5} \subset S_{5} \\
& C_{5} \subset S_{5} \\
& N_{5} \subset S_{5}
\end{aligned}
$$


Now, we can define the following functions that map the entire search space to boolean values indicating membership to the known set of splice sites.

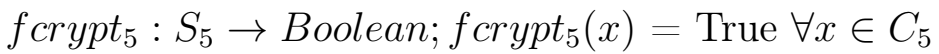

$$
\begin{aligned}
& \text { fauth }_{5}: S_{5} \rightarrow \text { Boolean; fauth } h_{5}(x)=\text { True } \forall x \in A_{5} \\
& \text { fsplice }_{5}: S_{5} \rightarrow \text { Boolean; } f \operatorname{splice}_{5}(x)=\text { True } \forall x \in\left\{A_{5} U C_{5}\right\}
\end{aligned}
$$

Similarly, for 3' splice sites, we can define the entire search space $S_{3}$ such that:

$$
\left|S_{3}\right|=4^{11}=4194304
$$

and we can define similar membership functions as follows:

$$
\begin{aligned}
& \text { frypt }_{3}: S_{3} \rightarrow \text { Boolean; frypt }{ }_{3}(x)=\text { True } \forall x \in C_{3} \\
& \text { fauth }_{3}: S_{3} \rightarrow \text { Boolean; fauth } \text { f }_{3}(x)=\text { True } \forall x \in A_{3}
\end{aligned}
$$

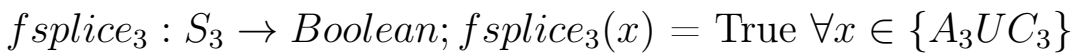

Now, we can redefine the problem statement defined in section 2 as follows:

Can each membership function on chromosomes (fcrypt, fauth and fsplice) be defined in terms of simpler functions of the individual genes?

We can use Genetic Algorithms to randomly extrapolate a subset of the search space $S_{5}$ with the help of a suitable fitness function. PWM is a widely used measure of the statistical distribution of nucleotides in an n-mer. Hence, the PWM score of an n-mer is used as the fitness function for the Genetic Algorithm. The extrapolated subset of $S_{5}$ should have a high degree of similarity to the dataset used to train the PWM. The similarity between datasets can be quantified using the scoring measures defined in the following subsections. 


\subsubsection{Computing exact match ratio (EMR)}

Let,

- $P W M_{-} A_{5}$ : PWM trained using $A_{5}$

- $P W M_{-} C_{5}$ : PWM trained using $C_{5}$

- $P W M_{-} N_{5}$ : PWM trained using $N_{5}$

- $E A_{5}$ : Subset of $S_{5}$ extrapolated using GA and $P W M_{-} A_{5}$

- $E C_{5}$ : Subset of $S_{5}$ extrapolated using GA and $P W M{ }_{-} C_{5}$

- $E N_{5}$ : Subset of $S_{5}$ extrapolated using GA and $P W M_{-} N_{5}$

We compare the $E A_{5}, E C_{5}$, and $E N_{5}$ subsets based on their degree of similarity to the subsets $A_{5}, C_{5}$, and $N_{5}$. For the sake of simplicity, we compute degree of similarity using exact match ratio (EMR).

Given two subsets S1 and S2, EMR can be defined as follows:

$$
\operatorname{EMR}(S 1, S 2)=|S 2 \cap S 1| /|S 2|
$$

For example, consider the following comparisons:

$\operatorname{EMR}\left(E A_{5}, A_{5}\right)=\left|A_{5} \cap E A_{5}\right| /\left|A_{5}\right|:$ Percentage of chromosomes in $A_{5}$ that are covered by $E A_{5}$

Similarly, we can define $\operatorname{EMR}\left(E A_{5}, C_{5}\right)=\left|C_{5} \cap E A_{5}\right| /\left|C_{5}\right|$

In simpler terms, $\operatorname{EMP}(\mathrm{S} 1, \mathrm{~S} 2)=$ ratio of individual n-mer strings in $\mathrm{S} 2$ covered by $\mathrm{S} 1$

\subsubsection{Competing datasets}

Two datasets are competing if they are supposed to be intrinsically different as per the hypothesis. Thus,

- $A_{5}$ competes $C_{5}$

- $C_{5}$ competes $A_{5}$ 
- $N_{5}$ competes $A_{5} \cup C_{5}$

\subsubsection{Comparison Scores}

Using the definition of EMR from equation-1, we can define the score metrics that quantify the degree of similarity for comparing the datasets.

Let us assume two datasets P and Q and their extrapolated sets EP and EQ (using GA with PWM). If $\mathrm{P}$ and $\mathrm{Q}$ are competing datasets (defined in section 5.2.4, we can define the following comparison scores:

- Preservation Score (PSCORE)

$$
\begin{aligned}
& P S C O R E(P)=E M R(E P, P) \\
& P S C O R E(Q)=E M R(E Q, Q)
\end{aligned}
$$

- Confusion Score (CSCORE)

$$
\begin{aligned}
& C S C O R E(P)=1-E M R(E P, Q) \\
& C \operatorname{CCORE}(Q)=1-E M R(E Q, P)
\end{aligned}
$$

If there exists a GA, that can give an EP and an EQ that maximize the scores PSCORE(P), PSCORE(Q), CSCORE(P), and $\operatorname{CSCORE}(\mathrm{Q})$, then we can conclude that $\mathrm{P}$ and $\mathrm{Q}$ are intrinsically different.

\subsubsection{Enhanced Scores}

The PSCORE and CSCORE measures are based on EMR, which looks for exact matches across the two participating datasets. The scores of the 3' splice sites are expected to be worse than the 5' splice sites because the 3' splice sites are longer and thus less likely to exactly match the known splice sites. Hence, the EMR based scores are too rigid for longer sequences. 
The coverage logic can be enhanced by considering local alignment scores instead of exact match. This should give us better coverage scores for 3' splice sites.

\subsubsection{Steps}

For 5' splice sites:

Given datasets: $A_{5}, C_{5}$, and $N_{5}$

1. Compute $P W M_{-} A_{5}, P W M_{-} C_{5}$, and $P W M_{-} N_{5}$ from $A_{5}, C_{5}$, and $N_{5}$ respectively

2. Initialize a random initial population $\mathrm{P}(1)$ of 9 -mers with the GT di-nucleotide at positions 4 and 5

3. Using $P W M_{-} A_{5}$ and a suitable GA parameters, extrapolate $E A_{5}$ from $\mathrm{P}(1)$

4. Using $P W M C_{5}$ and a suitable GA parameters, extrapolate $E C_{5}$ from $\mathrm{P}(1)$

5. Using $P W M_{-} N_{5}$ and a suitable GA parameters, extrapolate $E N_{5}$ from $\mathrm{P}(1)$

6. Compute PSCORE $\left(A_{5}\right)$ and $\operatorname{CSCORE}\left(A_{5}\right)$ given that $A_{5}$ and $C_{5}$ are competing

7. Compute PSCORE $\left(C_{5}\right)$ and $\operatorname{CSCORE}\left(C_{5}\right)$ given that $C_{5}$ and $A_{5}$ are competing

8. Compute PSCORE $\left(N_{5}\right)$ and $\operatorname{CSCORE}\left(N_{5}\right)$ given that $N_{5}$ and $A_{5} \cup C_{5}$ are competing

The steps for 3' splice sites are same as above. The given datasets for 3' splice sites are $A_{3}, C_{3}$, and $N_{3}$.

\subsubsection{Dataset Limitations}

For the sake of simplicity, the experiments presented in this report are performed on limited datasets that do not include all known splice sites. The datasets are specified in detail in section 3 . 


\subsection{Algorithms: Operator and Strategies}

\subsubsection{Selection Strategies}

1. Roulette Wheel Selection:

Example:

Table 5: Sample data: Roulette Wheel Selection

\begin{tabular}{|c|c|c|c|c|}
\hline Label & Chromosome & Fitness & Probability & Cumulative Probability \\
\hline \hline C1 & 1101010 & 30 & $30 / 80=0.375$ & 0.375 \\
\hline C2 & 1011001 & 15 & $15 / 80=0.1875$ & 0.5625 \\
\hline C3 & 0100110 & 10 & $10 / 80=0.125$ & 0.6875 \\
\hline C4 & 1000101 & 5 & $5 / 80=0.0625$ & 0.75 \\
\hline C5 & 1111000 & 20 & $20 / 80=0.25$ & 1 \\
\hline
\end{tabular}

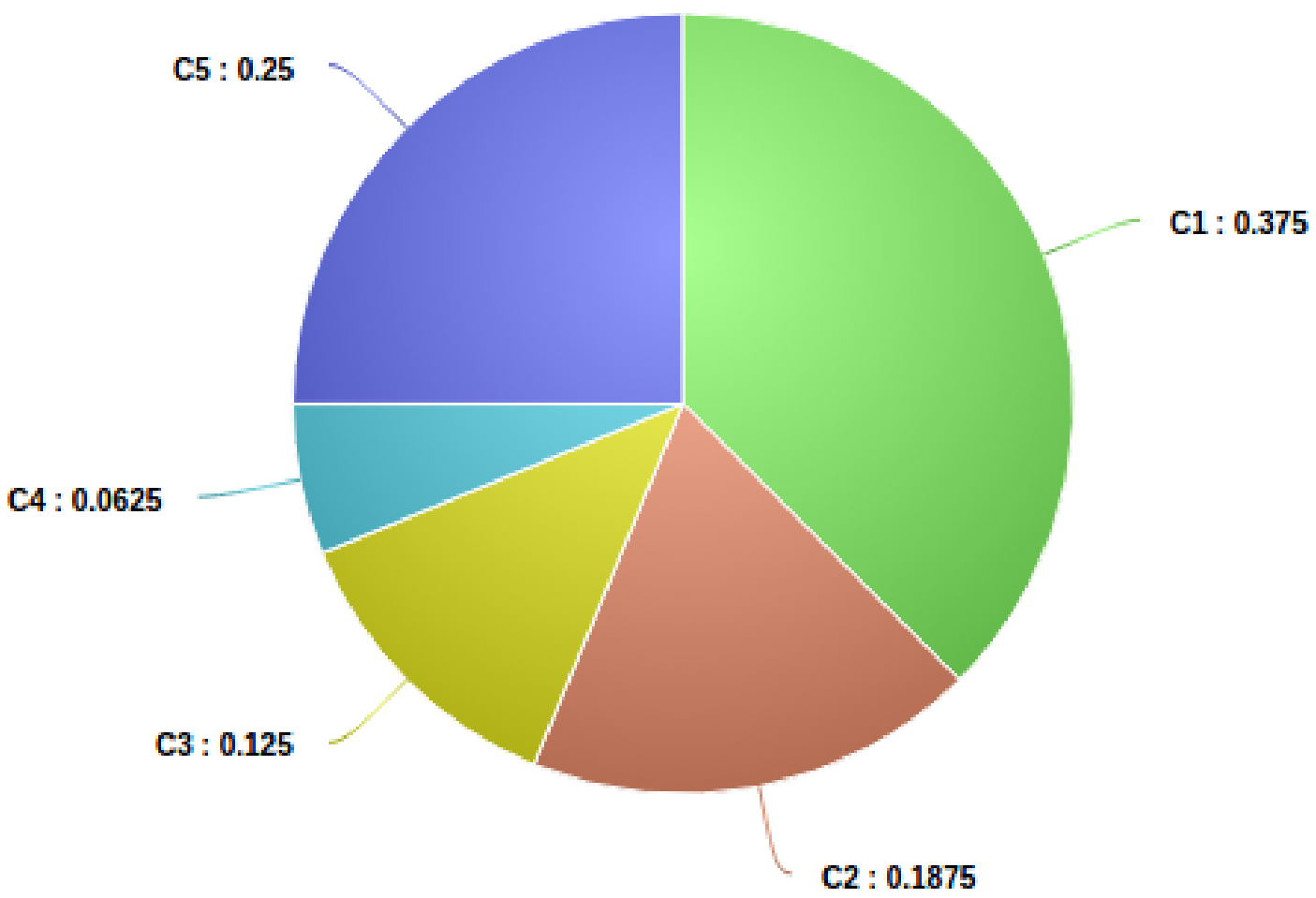

Figure 12: Roulette wheel for chromosomes in Table 5

\section{Algorithm:}


A random number generator is used to generate a value $r \epsilon[0,1]$. The cumulative probabilities are used to select a chromosome. For example, if $\mathrm{r}=0.7$, then $\mathrm{C} 4$ is selected since $0.6875<0.7<0.75$

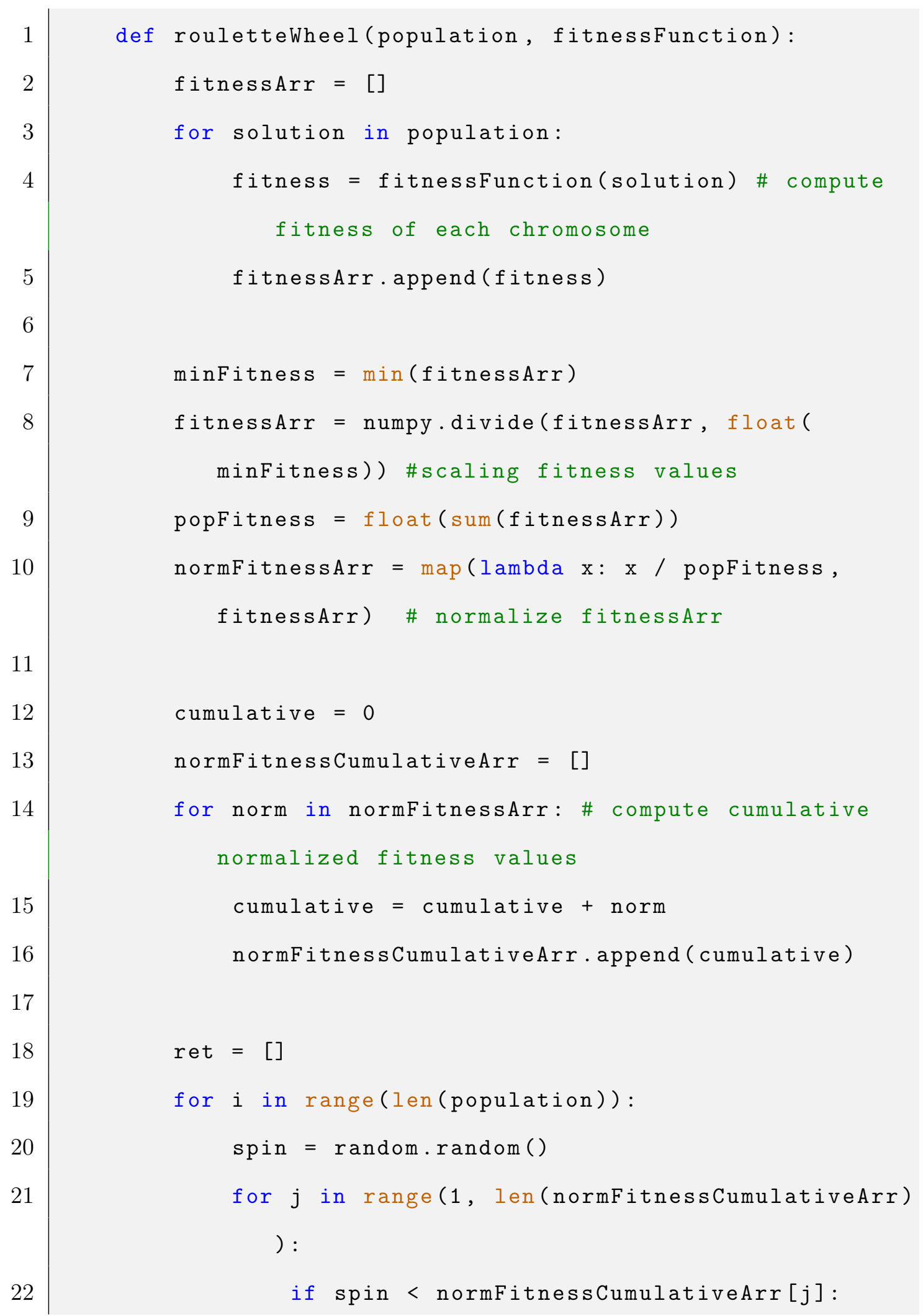




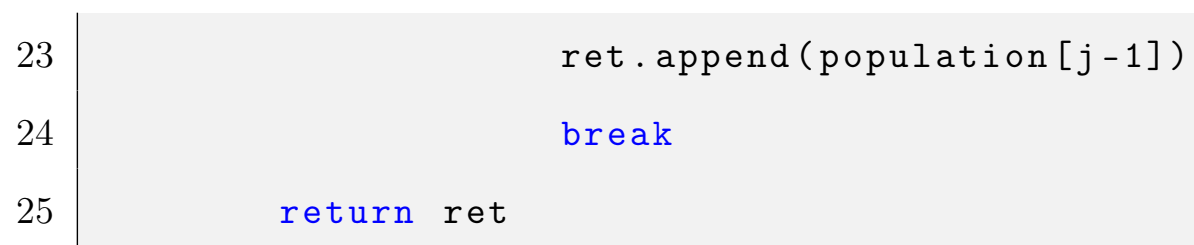

2. Ranked Selection:

Example:

Recomputing probabilities of the sample chromosomes from Table 5:

Table 6: Sample data: Ranked Selection

\begin{tabular}{|c|c|c|c|c|c|}
\hline Label & Chromosome & Fitness & Rank & Probability & Cumulative Probability \\
\hline \hline C1 & 1101010 & 30 & 5 & $5 / 15=0.333$ & 0.333 \\
\hline C2 & 1011001 & 15 & 3 & $3 / 15=0.2$ & 0.533 \\
\hline C3 & 0100110 & 10 & 2 & $2 / 15=0.133$ & 0.666 \\
\hline C4 & 1000101 & 5 & 1 & $1 / 15=0.067$ & 0.733 \\
\hline C5 & 1111000 & 20 & 4 & $4 / 15=0.267$ & 1 \\
\hline
\end{tabular}

\section{Algorithm:}

We can re-use the roulette wheel selection algorithm after recomputing the fitness probabilities as a linear function of the ranks.

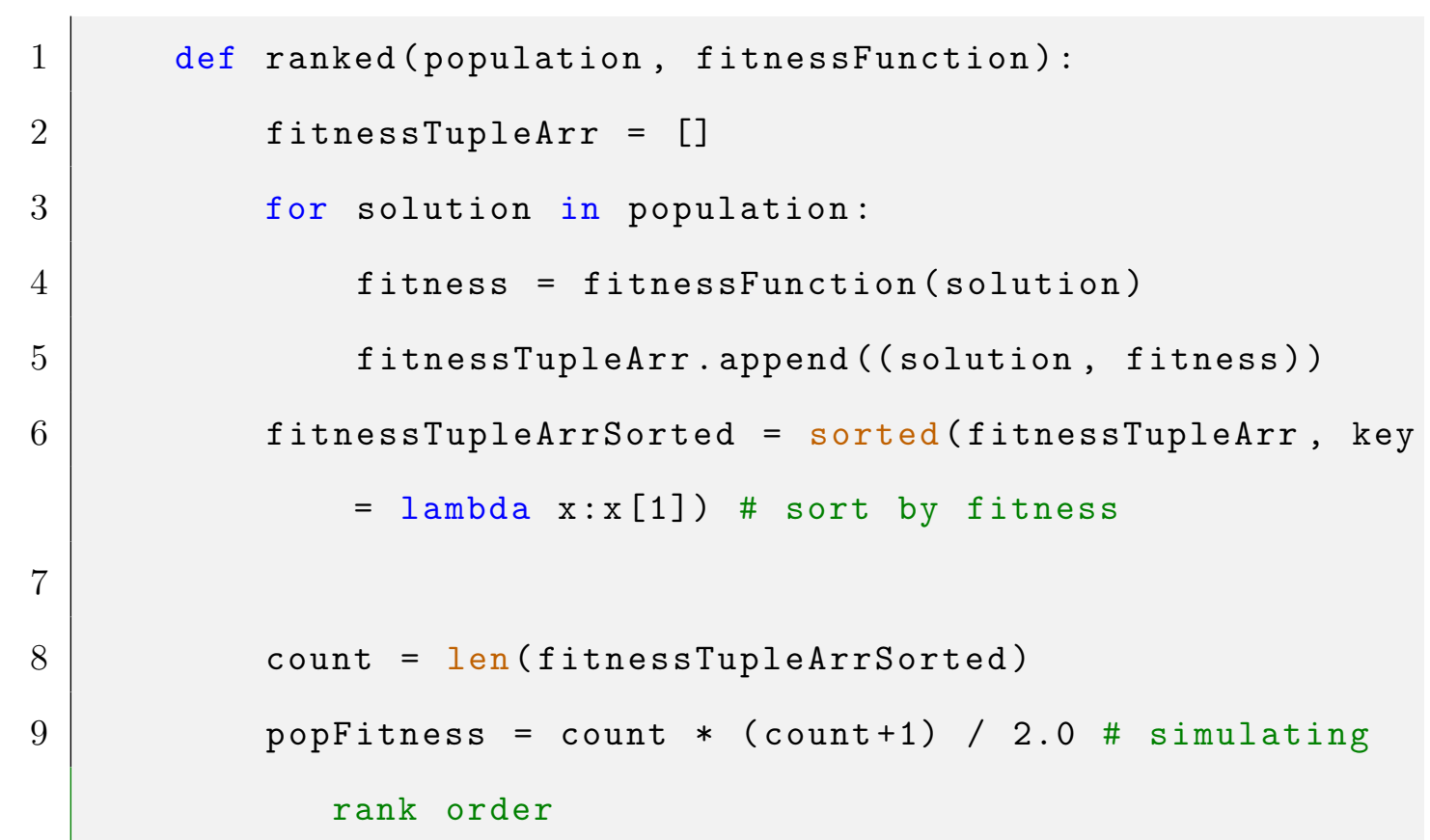




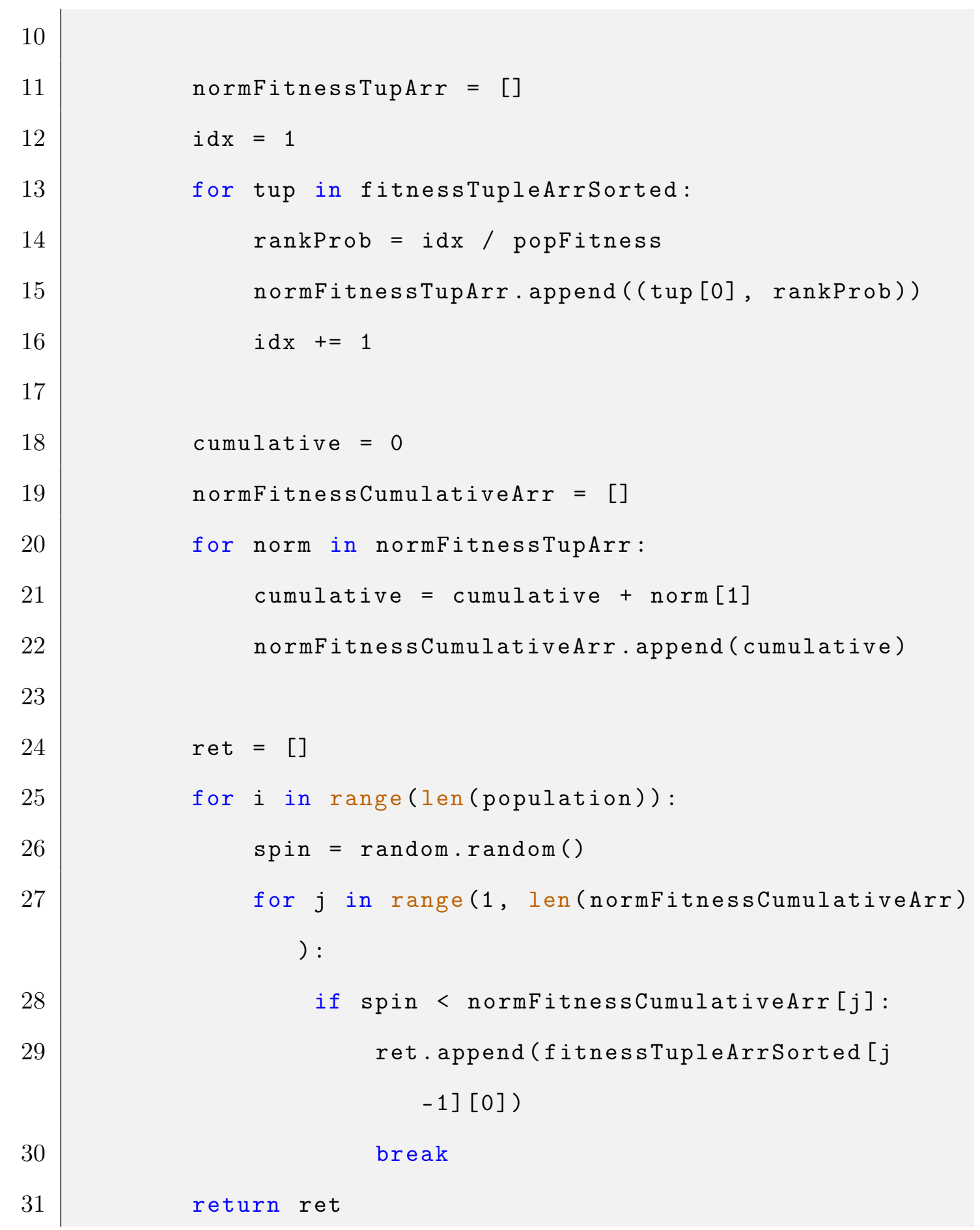

3. Tournament Selection:

\section{Algorithm:}

A fixed number (s) of random chromosomes are selected for a tournament. The chromosome with the highest fitness value among the s chromosomes is added to the mating pool. This process is repeated M times to select M chromosomes. 


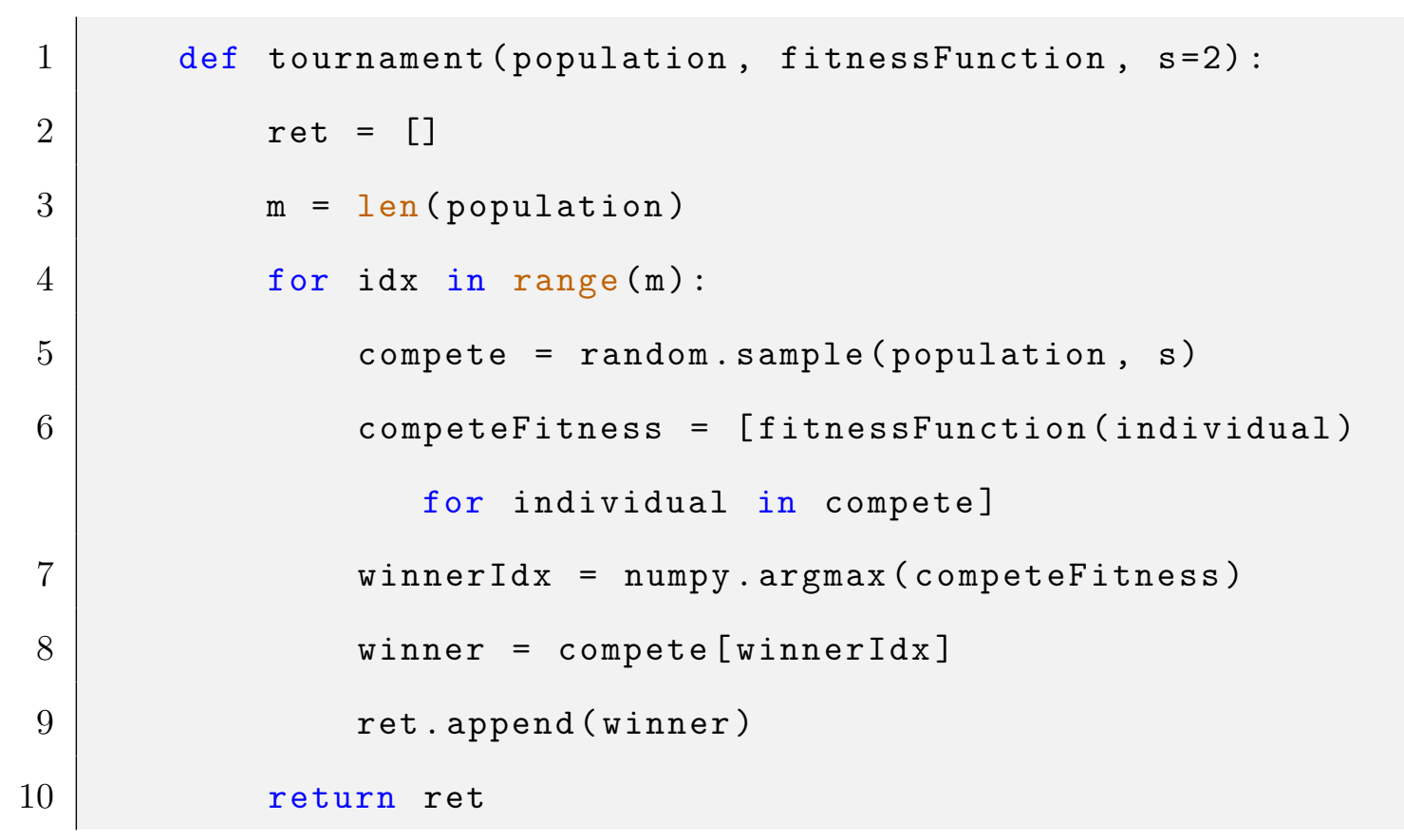

\subsubsection{Crossover Operators}

1. One Point Crossover:

Example:

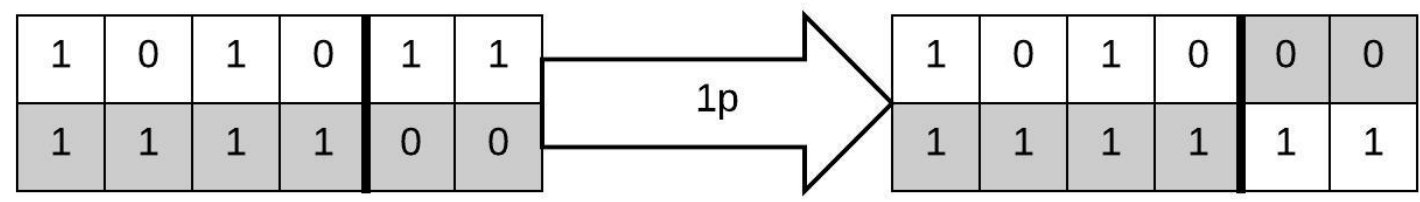

Figure 13: Example: One Point Crossover

\section{Algorithm:}

A crossover site is selected at random and all alleles on one side of the site are exchanged between the two parents.

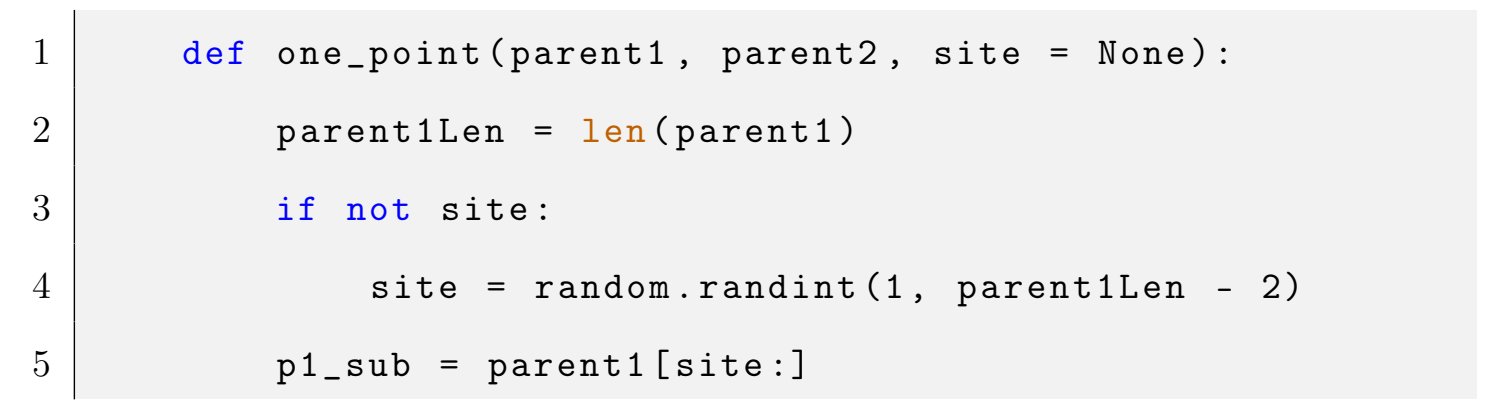




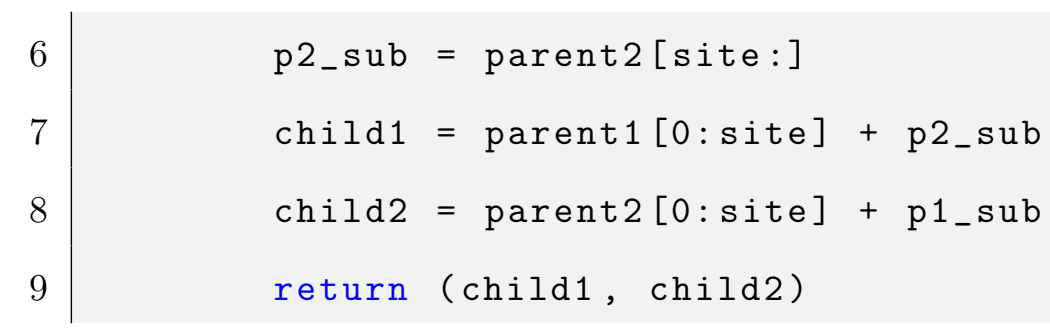

2. Two Point Crossover:

Example:

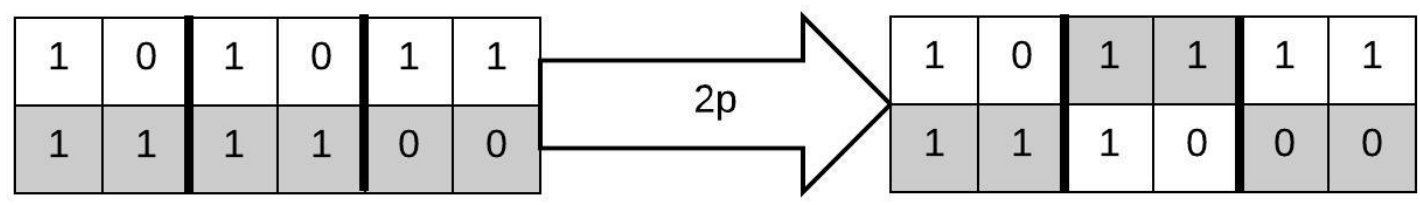

Figure 14: Example: Two Point Crossover

\section{Algorithm:}

Two crossover sites are selected at random and all alleles between the two sites are exchanged between the two parents.

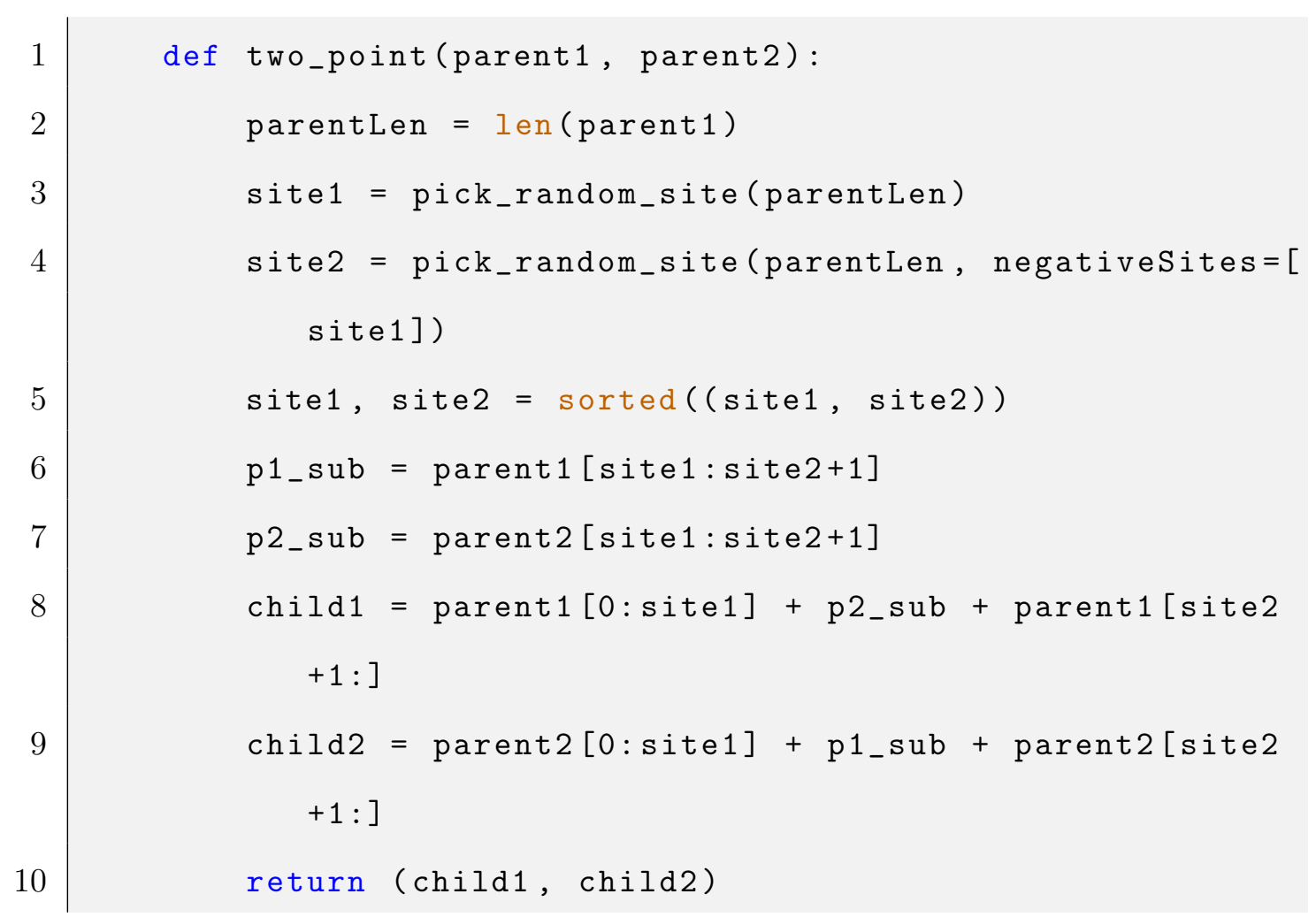


3. k-Point Crossover:

The concept of 1-point or 2-point crossover can be extended to k points. The alleles between each odd crossover point and its immediately successive even crossover point are exchanged between the two parents.

4. Uniform Crossover:

Example:

\begin{tabular}{|c|c|c|c|c|c|c|c|c|c|c|c|c|}
\hline 1 & 0 & 1 & 0 & 1 & 1 & & 1 & 1 & 1 & 0 & 1 & 0 \\
\hline 1 & 1 & 1 & 1 & 0 & 0 & 19 & 1 & 0 & 1 & 1 & 0 & 1 \\
\hline 0.2 & 0.7 & 0.8 & 0.1 & 0.3 & 0.6 & & & & & & & \\
\hline
\end{tabular}

Figure 15: Example: Uniform Crossover

\section{Algorithm:}

Each gene in the chromosome is considered for crossover with some swapping probability $p_{e}$. The alleles at the chosen gene locations $\left(R>p_{e}\right)$ are swapped between the two parents.

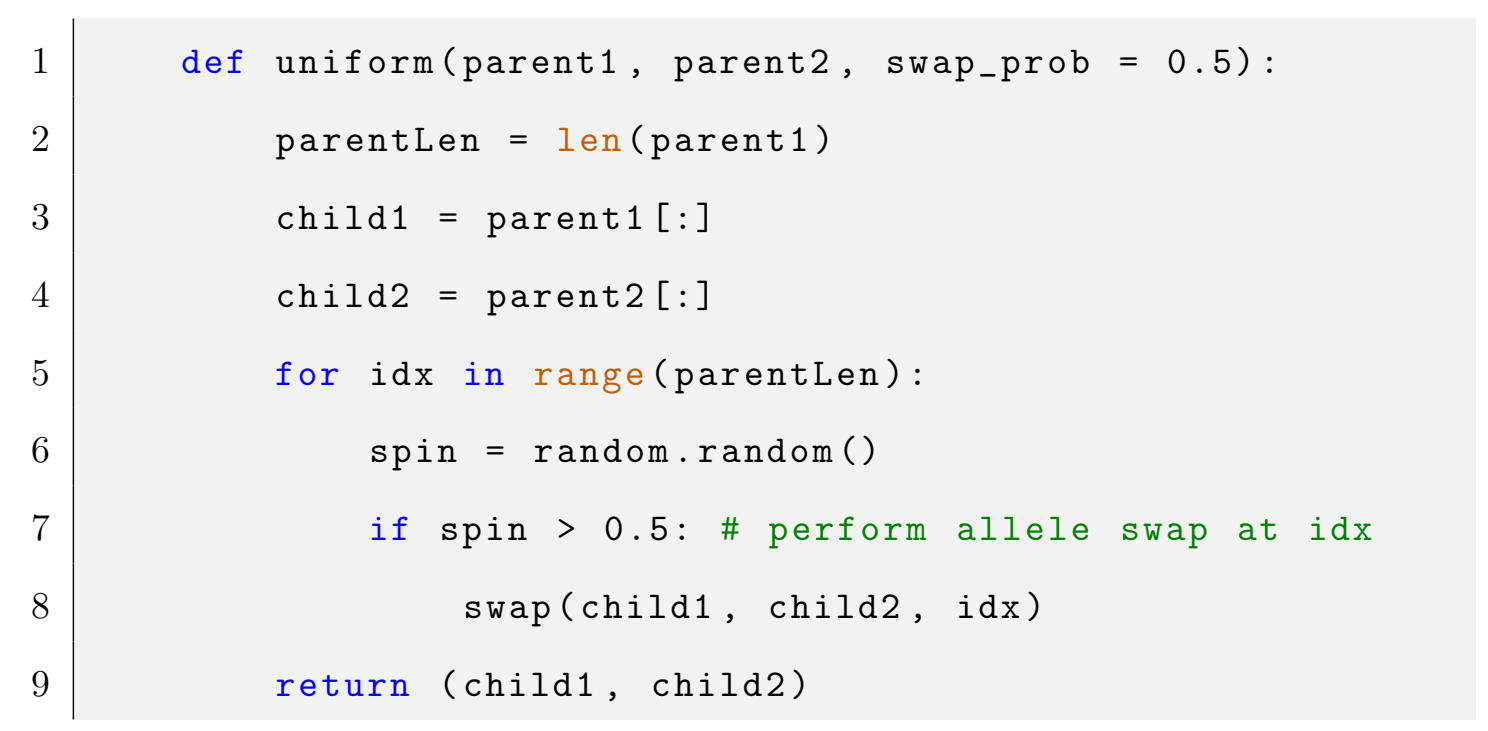

5. Uniform Order-Based Crossover: Example:

\section{Algorithm:}




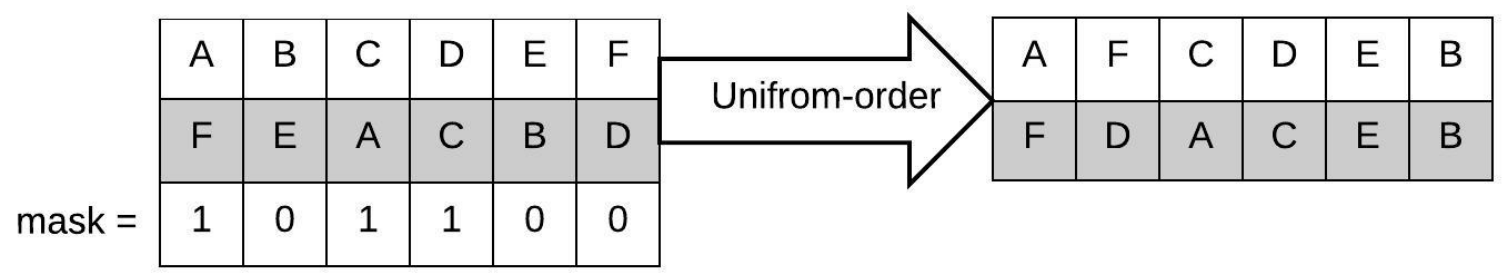

Figure 16: Example: Uniform Order-Based Crossover

A random binary template is used to guide the crossover. The alleles at indices in the parents corresponding to a 0 in the mask are reordered according to their order in the other chromosome.

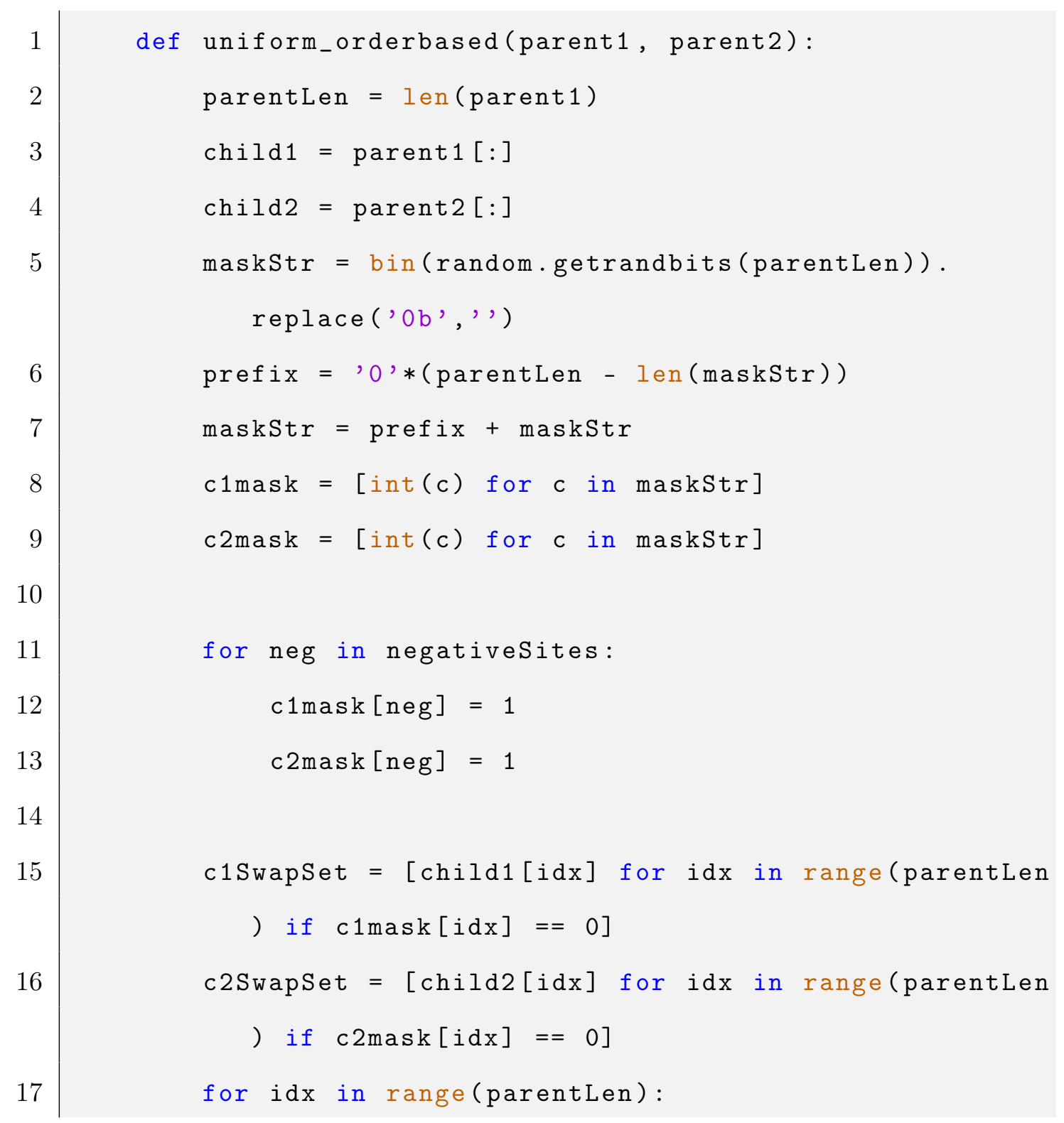




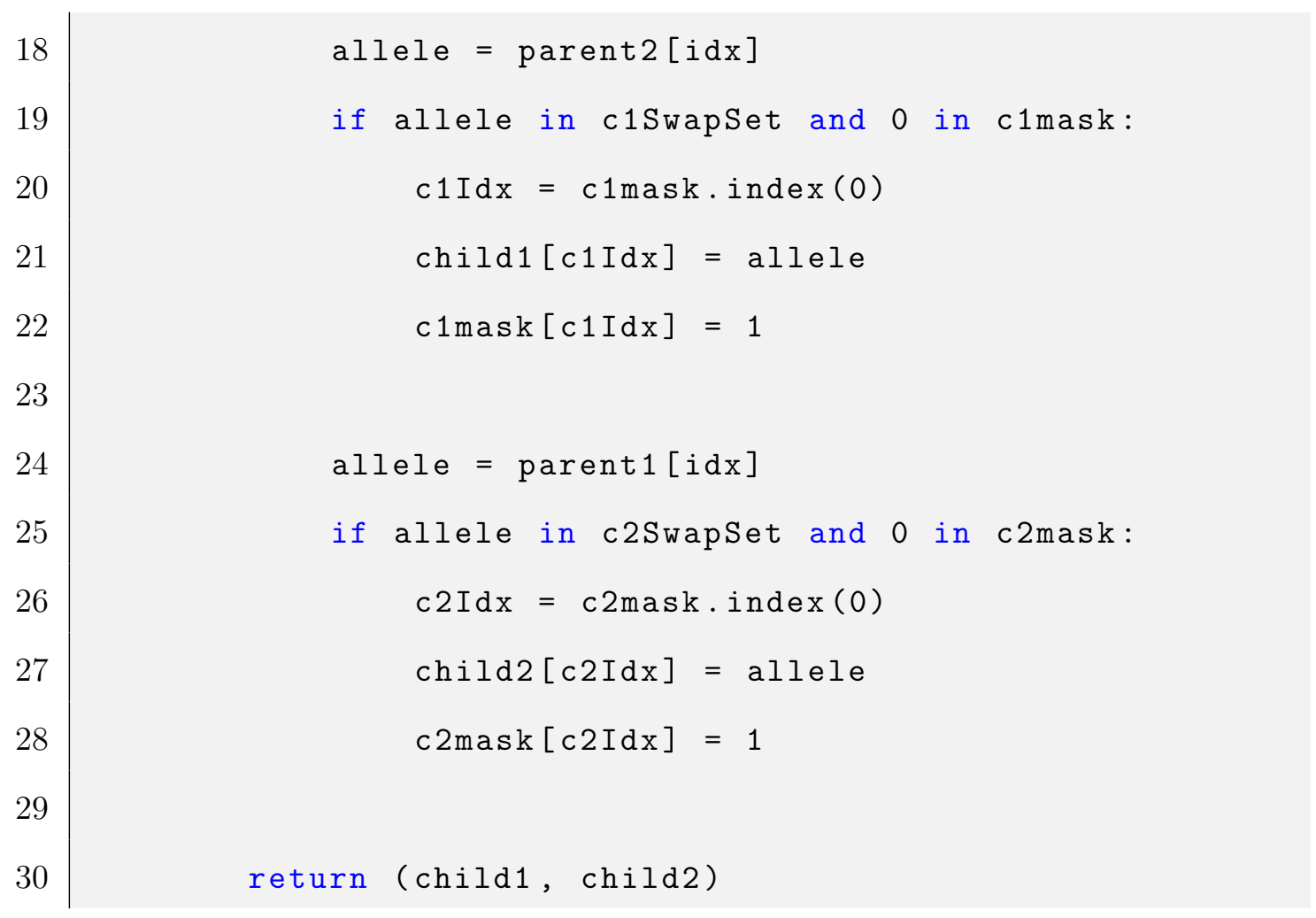

All the operator and strategy algorithms specified in this section can be plugged into the parametrized GA framework defined in figure ??. The next section presents the results of executing the GA framework for each combination of the operators and strategies. Since the result of one execution of a parametrized GA is stochastic, each instance of the parametrized GA is executed hundred times.

\section{$6 \quad$ Results}

The algorithm defined in steps section 5.2.7 was executed for each combination of the following parameters for GA:

- generation count: $\{10,100,1000\}$ - number of generations

- generation size: $\{10,20\}$ - size of each generation

- selection strategy: \{roulette wheel, ranked, tournament\}

- crossover operator: \{one point, two point, uniform, uniform order based\} 


\subsection{Scoring Tables}

For the set of parameter values defined above, there are 3 X 2 X 3 X $4=72$ parameter combinations. Each combination is executed 100 times in order to account for the stochastic nature of the results of each execution.

The format of the scoring tables is as follows:

- Title of the table indicates the selection strategy and the crossover operator

- Rows 1 - 3 indicate the dataset (AUTH, CSS, or NEIGHBOR), the scoring metric (PSCORE, CSCORE or FITNESS), and the GA parameters m (generation size) and gc (generation count)

- For a single set of parameter values for $\mathrm{m}$ and gc, there is a composite column comprising of two subcolumns $\mathrm{f}(\bar{x})$ and $\mathrm{N}$.

$\mathrm{f}(\bar{x})=$ unique value of the score metric

$\mathrm{N}=$ frequency of the $\mathrm{f}(\bar{x})$ value across 100 execution

- The last row contains the mean of the scoring metric across all 100 execution

- Row 5 to second last row contain the top eight $\mathrm{f}(\bar{x})$ values based on their frequency across the 100 executions

The scoring tables for the best performing parameter combination for each dataset with respect to the PSCORE, CSCORE, and FITNESS are listed in this section. 


\subsection{Scoring Tables: 5'}

Table 7: SELECTION: ROULETTEWHEEL; CROSSOVER: 2P: AUTH - CSCORE

\begin{tabular}{|c|c|c|c|c|c|c|c|c|c|c|c|}
\hline \multicolumn{12}{|c|}{ AUTH - CSCORE } \\
\hline \multicolumn{2}{|c|}{ AUTH-10 } & \multicolumn{2}{|c|}{ AUTH-10 } & \multicolumn{2}{|c|}{ AUTH-10 } & \multicolumn{2}{|c|}{ AUTH-20 } & \multicolumn{2}{|c|}{ AUTH-20 } & \multicolumn{2}{|c|}{ AUTH-20 } \\
\hline \multicolumn{2}{|c|}{$\mathrm{m}=10, \mathrm{gc}=10$} & \multicolumn{2}{|c|}{$\mathrm{m}=10, \mathrm{gc}=100$} & \multicolumn{2}{|c|}{$\mathrm{m}=10, \mathrm{gc}=1000$} & \multicolumn{2}{|c|}{$\mathrm{m}=20, \mathrm{gc}=10$} & \multicolumn{2}{|c|}{$\mathrm{m}=20, \mathrm{gc}=100$} & \multicolumn{2}{|c|}{$\mathrm{m}=20, \mathrm{gc}=1000$} \\
\hline $\mathrm{f}(\bar{x})$ & $\mathrm{N}$ & $\mathrm{f}(\bar{x})$ & $\mathrm{N}$ & $\mathrm{f}(\bar{x})$ & $\mathrm{N}$ & $\mathrm{f}(\bar{x})$ & $\mathrm{N}$ & $\mathrm{f}(\bar{x})$ & $\mathrm{N}$ & $\mathrm{f}(\bar{x})$ & $\mathrm{N}$ \\
\hline 1.0 & 100 & 1.0 & 84 & 1.0 & 42 & 1.0 & 97 & 1.0 & 89 & 1.0 & 63 \\
\hline \multirow[t]{8}{*}{1.0} & 100 & 1.0 & 84 & 1.0 & 42 & 1.0 & 97 & 1.0 & 89 & 1.0 & 63 \\
\hline & & 0.4 & 2 & 1.0 & 42 & 0.95 & 3 & 0.9 & 1 & 1.0 & 63 \\
\hline & & 0.8 & 1 & 0.1 & 5 & & & 0.8 & 1 & 0.55 & 2 \\
\hline & & 0.6 & 1 & 0.4 & 8 & & & 0.95 & 9 & 0.8 & 3 \\
\hline & & 0.9 & 11 & 0.2 & 1 & & & & & 0.4 & 1 \\
\hline & & 0.7 & 1 & 0.3 & 1 & & & & & 0.65 & 1 \\
\hline & & & & 0.6 & 5 & & & & & 0.85 & 2 \\
\hline & & & & 0.8 & 6 & & & & & 0.1 & 1 \\
\hline \multicolumn{2}{|c|}{$\hat{\mathrm{f}}=1.0$} & \multicolumn{2}{|c|}{$\hat{\mathrm{f}}=0.968$} & \multicolumn{2}{|c|}{$\hat{\mathrm{f}}=0.779$} & \multicolumn{2}{|c|}{$\hat{\mathrm{f}}=0.9985$} & \multicolumn{2}{|c|}{$\hat{\mathrm{f}}=0.9925$} & \multicolumn{2}{|c|}{$\hat{\mathrm{f}}=0.925$} \\
\hline
\end{tabular}

The scores specified in Table-7 are obtained by executing a GA with roulette wheel selection and two point crossover on authentic 5' splice sites for each combination of generation size $(\mathrm{m})$ and generation count $(\mathrm{gc})$. The score function is the confusion score defined in equation-4. As defined in section-5.2.4, the competing dataset for authentic 5' splice sites $\left(A_{5}\right)$ is the cryptic 5' splice sites $\left(C_{5}\right)$. Hence, confusion score is a measure of the dissimilarity between authentic and cryptic 5' splice sites.

From table-7, we can interpret the results for each generation size(m) and generation count $(\mathrm{gc})$ as follows:

- For $\mathrm{m}=10, \mathrm{gc}=10$, the mean score $\hat{\mathrm{f}}$ of 1.0 indicates that there is zero percent match between the extrapolated authentic splice sites and the known cryptic splice sites. Although this result is favorable to the hypothesis, it is not a useful result because of the small size of the datasets being compared using cscore.

- For a given $\mathrm{m}$, the mean CSCORE decreases as the number generations increases. This indicates that as the generations progress, some of the known cryptic splice sites start appearing in the extrapolated authentic splice sites. 
- For a given $\operatorname{gc}(10,100$, or 1000$)$, the score with the higher generation $\operatorname{size}(\mathrm{m}=20)$ is better than the lower generation $\operatorname{size}(\mathrm{m}=10)$.

- Based on the frequency of cscore distribution across the hundred executions, $\mathrm{m}=20$ with $\mathrm{gc}=100$ shows the best results. Out of the hundred executions, 89 of them reported a high cscore of $1.0,9$ executions reported cscore $=0.95$, while two executions reported 0.9 and 0.8 each.

- Although $\mathrm{m}=20$ with $\mathrm{gc}=1000$ has a close mean score to $\mathrm{gc}=100$, it shows a much wider distribution of the cscore across the hundred executions. Thus, the parameters $\mathrm{m}=20$ with $\mathrm{gc}=100$ report the best cscore for authentic 5' splice sites.

Table 8: SELECTION: ROULETTEWHEEL; CROSSOVER: UNIFORMORDERBASED: CSS - FITNESS

\begin{tabular}{|c|c|c|c|c|c|c|c|c|c|c|c|}
\hline \multicolumn{12}{|c|}{ CSS - fitness } \\
\hline \multicolumn{2}{|l|}{ CSS-10 } & \multicolumn{2}{|l|}{ CSS-10 } & \multicolumn{2}{|l|}{ CSS-10 } & \multicolumn{2}{|l|}{ CSS-20 } & \multicolumn{2}{|l|}{ CSS-20 } & \multicolumn{2}{|l|}{ CSS-20 } \\
\hline \multicolumn{2}{|c|}{$\mathrm{m}=10, \mathrm{gc}=10$} & \multicolumn{2}{|c|}{$\mathrm{m}=10, \mathrm{gc}=100$} & \multicolumn{2}{|c|}{$\mathrm{m}=10, \mathrm{gc}=1000$} & \multicolumn{2}{|c|}{$\mathrm{m}=20, \mathrm{gc}=10$} & \multicolumn{2}{|c|}{$\mathrm{m}=20, \mathrm{gc}=100$} & \multicolumn{2}{|c|}{$\mathrm{m}=20, \mathrm{gc}=1000$} \\
\hline $\mathrm{f}(\bar{x})$ & $\mathrm{N}$ & $\mathrm{f}(\bar{x})$ & $\mathrm{N}$ & $\mathrm{f}(\bar{x})$ & $\mathrm{N}$ & $\mathrm{f}(\bar{x})$ & $\mathrm{N}$ & $\mathrm{f}(\bar{x})$ & $\mathrm{N}$ & $\mathrm{f}(\bar{x})$ & $\mathrm{N}$ \\
\hline 10.2745848678 & 7 & 9.6636271585 & 17 & 10.5546927869 & 28 & 7.86063569485 & 7 & 9.79887986855 & 8 & 9.79887986855 & 24 \\
\hline 10.2745848678 & 7 & 9.6636271585 & 17 & 10.5546927869 & 28 & 7.86063569485 & 7 & 9.79887986855 & 8 & 9.79887986855 & 24 \\
\hline 9.52939876679 & 2 & 10.2745848678 & 15 & 10.2745848678 & 13 & 7.47156717343 & 1 & 9.32501268826 & 1 & 9.25645354096 & 1 \\
\hline 7.09569742324 & 1 & 9.67078985187 & 2 & 9.83010359059 & 1 & 8.57170792736 & 1 & 6.83397962429 & 1 & 8.27497200576 & 1 \\
\hline 6.74569466089 & 1 & 9.17550294324 & 3 & 10.4204643952 & 3 & 6.31901440884 & 1 & 8.27497200576 & 1 & 9.6636271585 & 1 \\
\hline 7.54206517862 & 1 & 7.67762193286 & 1 & 9.79887986855 & 4 & 10.2745848678 & 3 & 9.93310826026 & 1 & 8.15737161645 & 1 \\
\hline 7.03503517961 & 1 & 7.76382598768 & 2 & 7.97163643169 & 1 & 5.62964325793 & 1 & 7.85805135572 & 1 & 10.2745848678 & 4 \\
\hline 10.2745848678 & 7 & 8.31388111948 & 1 & 7.73996605529 & 1 & 8.79061043701 & 1 & 9.67078985187 & 1 & 6.12665944794 & 1 \\
\hline 6.49190240736 & 1 & 6.60836516393 & 1 & 8.69979794403 & 1 & 9.6789085118 & 1 & 3.07984021931 & 1 & 9.17550294324 & 1 \\
\hline \multicolumn{2}{|c|}{$\hat{\mathrm{f}}=8.17125686486$} & \multicolumn{2}{|c|}{$\hat{\mathrm{f}}=8.90459883956$} & \multicolumn{2}{|c|}{$\hat{\mathrm{f}}=9.83882318289$} & \multicolumn{2}{|c|}{$\hat{\mathrm{f}}=7.85153157327$} & \multicolumn{2}{|c|}{$\hat{\mathrm{f}}=8.47527857503$} & \multicolumn{2}{|c|}{$\hat{\mathrm{f}}=9.03385109483$} \\
\hline
\end{tabular}

The scores specified in Table- 8 are obtained by executing a GA with roulette wheel selection and uniform ordered crossover on cryptic 5' splice sites for the same combinations of generation size $(\mathrm{m})$ and generation count $(\mathrm{gc})$. The score function is the PWM score fitness as defined in section-5.2.1.

- For a given $\mathrm{m}$, the mean fitness $\hat{\mathrm{f}}$ increases as the number generations increases. This indicates that, as the generations progress, the population converges towards 
healthier individuals overall. This can be justified using the Schema theorem [12].

- For a given $\operatorname{gc}(10,100$, or 1000), the score with the higher generation $\operatorname{size}(\mathrm{m}=$ $20)$ is worse than the lower generation $\operatorname{size}(m=10)$. This indicates that the best fitness value that a population could achieve in this case, decreased as the number of individuals in the population increased.

- Based on the frequency of fitness distribution across the hundred executions, $\mathrm{m}=10$ with $\mathrm{gc}=1000$ shows the best results. Out of the hundred executions, 28 of them reported the highest PWM score fitness of 10.55 .

Table 9: SELECTION: ROULETTEWHEEL; CROSSOVER: UNIFORMORDERBASED: NEIGHBOR - CSCORE

\begin{tabular}{|c|c|c|c|c|c|c|c|c|c|c|c|}
\hline \multicolumn{12}{|c|}{ NEIGHBOR - compete } \\
\hline \multicolumn{2}{|c|}{ NEIGHBOR-10 } & \multicolumn{2}{|c|}{ NEIGHBOR-10 } & \multicolumn{2}{|c|}{ NEIGHBOR-10 } & \multicolumn{2}{|c|}{ NEIGHBOR-20 } & \multicolumn{2}{|c|}{ NEIGHBOR-20 } & \multicolumn{2}{|c|}{ NEIGHBOR-20 } \\
\hline \multicolumn{2}{|c|}{$\mathrm{m}=10, \mathrm{gc}=10$} & \multicolumn{2}{|c|}{$\mathrm{m}=10, \mathrm{gc}=100$} & \multicolumn{2}{|c|}{$\mathrm{m}=10, \mathrm{gc}=1000$} & \multicolumn{2}{|c|}{$\mathrm{m}=20, \mathrm{gc}=10$} & \multicolumn{2}{|c|}{$\mathrm{m}=20, \mathrm{gc}=100$} & \multicolumn{2}{|c|}{$\mathrm{m}=20, \mathrm{gc}=1000$} \\
\hline $\mathrm{f}(\bar{x})$ & $\mathrm{N}$ & $\mathrm{f}(\bar{x})$ & $\mathrm{N}$ & $\mathrm{f}(\bar{x})$ & $\mathrm{N}$ & $\mathrm{f}(\bar{x})$ & $\mathrm{N}$ & $\mathrm{f}(\bar{x})$ & $\mathrm{N}$ & $\mathrm{f}(\bar{x})$ & $\mathrm{N}$ \\
\hline 1.0 & 50 & 1.0 & 38 & 1.0 & 41 & 0.95 & 30 & 0.95 & 23 & 1.0 & 26 \\
\hline 1.0 & 50 & 1.0 & 38 & 1.0 & 41 & 0.95 & 30 & 0.95 & 23 & 1.0 & 26 \\
\hline 1.0 & 50 & 1.0 & 38 & 1.0 & 41 & 1.0 & 22 & 1.0 & 22 & 1.0 & 26 \\
\hline 0.4 & 3 & 0.4 & 3 & 0.4 & 1 & 0.85 & 13 & 0.55 & 1 & 0.6 & 3 \\
\hline 0.8 & 15 & 0.8 & 18 & 0.8 & 7 & 0.55 & 1 & 0.8 & 8 & 0.55 & 1 \\
\hline 0.6 & 6 & 0.1 & 1 & 0.3 & 1 & 0.8 & 5 & 0.6 & 1 & 0.8 & 4 \\
\hline 0.3 & 2 & 0.6 & 7 & 0.6 & 6 & 0.9 & 17 & 0.85 & 11 & 0.9 & 18 \\
\hline 0.9 & 20 & 0.2 & 1 & 0.9 & 27 & 0.6 & 2 & 0.9 & 18 & 0.85 & 13 \\
\hline 0.7 & 3 & 0.9 & 20 & 0.7 & 10 & 0.65 & 2 & 0.7 & 7 & 0.4 & 1 \\
\hline \multicolumn{2}{|c|}{$\hat{\mathrm{f}}=0.88$} & \multicolumn{2}{|c|}{$\hat{\mathrm{f}}=0.841$} & \multicolumn{2}{|c|}{$\hat{\mathrm{f}}=0.857$} & \multicolumn{2}{|c|}{$\hat{\mathrm{f}}=0.8955$} & \multicolumn{2}{|c|}{$\hat{\mathrm{f}}=0.884$} & \multicolumn{2}{|c|}{$\hat{\mathrm{f}}=0.8895$} \\
\hline
\end{tabular}

The scores specified in Table-9 are obtained by executing a GA with roulette wheel selection and uniform ordered crossover on Neighboring 5' splice sites. The score function is the confusion score defined in equation-4. As defined in section-5.2.4, the competing dataset for neighboring 5' splice sites $\left(N_{5}\right)$ is the union of cryptic and authentic 5' splice $\operatorname{sites}\left(A_{5} \cup C_{5}\right)$. The observations here are similar to the cscore results from Table- 7 .

- For a given $\mathrm{m}$, the mean cscore decreases as the number generations increases. This indicates that, as the generations progress, some of the known splice sites 
start appearing in the extrapolated neighboring putative splice sites.

- For a given $\operatorname{gc}(10,100$, or 1000$)$, the score with the higher generation $\operatorname{size}(\mathrm{m}=20)$ is better than the lower generation $\operatorname{size}(\mathrm{m}=10)$ except for $\mathrm{gc}=1000$.

- Based on the frequency of cscore distribution across the hundred exections, $\mathrm{m}=10$ with $\mathrm{gc}=1009$ shows the best results. Out of the hundred executions, 41 of them reported a high cscore of 1.0.

\subsection{Scoring Tables: 3'}

Table 10: SELECTION: ROULETTEWHEEL; CROSSOVER: UNIFORMORDERBASED: AUTH - FITNESS

\begin{tabular}{|c|c|c|c|c|c|c|c|c|c|c|c|}
\hline \multicolumn{12}{|c|}{ AUTH - fitness } \\
\hline \multicolumn{2}{|l|}{ AUTH-10 } & \multicolumn{2}{|l|}{ AUTH-10 } & \multicolumn{2}{|l|}{ AUTH-10 } & \multicolumn{2}{|l|}{ AUTH-20 } & \multicolumn{2}{|l|}{ AUTH-20 } & \multicolumn{2}{|l|}{ AUTH-20 } \\
\hline \multicolumn{2}{|c|}{$\mathrm{m}=10, \mathrm{gc}=10$} & \multicolumn{2}{|c|}{$\mathrm{m}=10, \mathrm{gc}=100$} & \multicolumn{2}{|c|}{$\mathrm{m}=10, \mathrm{gc}=1000$} & \multicolumn{2}{|c|}{$\mathrm{m}=20, \mathrm{gc}=10$} & \multicolumn{2}{|c|}{$\mathrm{m}=20, \mathrm{gc}=100$} & \multicolumn{2}{|c|}{$\mathrm{m}=20, \mathrm{gc}=1000$} \\
\hline $\mathrm{f}(\bar{x})$ & $\mathrm{N}$ & $\mathrm{f}(\bar{x})$ & $\mathrm{N}$ & $\mathrm{f}(\bar{x})$ & $\mathrm{N}$ & $\mathrm{f}(\bar{x})$ & $\mathrm{N}$ & $\mathrm{f}(\bar{x})$ & $\mathrm{N}$ & $\mathrm{f}(\bar{x})$ & $\mathrm{N}$ \\
\hline 6.3310505573 & 7 & 14.4612478686 & 48 & 14.4612478686 & 51 & 5.60441620002 & 11 & 5.60441620002 & 7 & 11.6082184986 & 5 \\
\hline 6.3310505573 & 7 & 14.4612478686 & 48 & 14.4612478686 & 51 & 5.60441620002 & 11 & 5.60441620002 & 7 & 11.6082184986 & 5 \\
\hline 12.7507896657 & 1 & 10.9189661574 & 1 & 7.12744429663 & 1 & -0.312763542694 & 1 & 3.6195612137 & 1 & 2.78157522191 & 1 \\
\hline 8.74171451452 & 1 & 13.5338392434 & 1 & 8.52921778081 & 1 & 4.35820017723 & 1 & 2.18544963501 & 1 & 3.74587732303 & 1 \\
\hline 10.8756868439 & 1 & 10.3421469139 & 1 & 12.2253609616 & 1 & 5.60003674027 & 1 & 1.71773340113 & 1 & 3.18190872128 & 1 \\
\hline 12.175587014 & 1 & 11.2571484401 & 1 & 7.50321011761 & 1 & 1.89270261223 & 1 & -0.433157556824 & 1 & 2.21196853795 & 1 \\
\hline 10.3886531907 & 1 & 10.6611347935 & 1 & 12.822616703 & 1 & 6.44238486089 & 1 & 2.60414034248 & 1 & 2.9424802076 & 1 \\
\hline 13.9105076821 & 2 & 9.99674996565 & 1 & 9.2834903204 & 1 & 4.78738801701 & 1 & 7.97827352522 & 1 & 1.77085644407 & 1 \\
\hline 7.03896738342 & 1 & 6.99678760007 & 1 & 12.47465425 & 1 & 1.60136799488 & 1 & 4.87697425919 & 1 & 3.72888579633 & 1 \\
\hline \multicolumn{2}{|c|}{$\hat{\mathrm{f}}=9.84240719451$} & \multicolumn{2}{|c|}{$\hat{\mathrm{f}}=12.4655497918$} & \multicolumn{2}{|c|}{$\hat{\mathrm{f}}=13.1848227782$} & \multicolumn{2}{|c|}{$\hat{\mathrm{f}}=3.67295265604$} & \multicolumn{2}{|c|}{$\hat{\mathrm{f}}=4.30809631847$} & \multicolumn{2}{|c|}{$\hat{\mathrm{f}}=4.53069290039$} \\
\hline
\end{tabular}

The scores specified in Table-10 are obtained by executing a GA with roulette wheel selection and uniform ordered crossover on authentic 3' splice sites for each combination of generation size $(\mathrm{m})$ and generation $\operatorname{count}(\mathrm{gc})$. The score function is the PWM score fitness as defined in section-5.2.1.

From table-10, we can interpret the results for each generation size(m) and generation count (gc) as follows: 
- For a given $\mathrm{m}$, the mean fitness $\hat{\mathrm{f}}$ increases as the number generations increases. This observation is similar to Table- 8 .

- For a given $\operatorname{gc}(10,100$, or 1000$)$, the score with the higher generation $\operatorname{size}(\mathrm{m}=20)$ is worse than the lower generation $\operatorname{size}(\mathrm{m}=10)$. However, compared to Table- 8 , the reduction in fitness is significantly more.

- Based on the frequency of fitness distribution across the hundred executions, $\mathrm{m}=10$ gives better results than $m=20$. Thus, the parameters shown this table lead to different results as compared to other parameters.

Table 11: SELECTION: TOURNAMENT; CROSSOVER: 1P: CSS - CSCORE

\begin{tabular}{|c|c|c|c|c|c|c|c|c|c|c|c|}
\hline \multicolumn{12}{|c|}{ CSS - compete } \\
\hline \multicolumn{2}{|c|}{ CSS-10 } & \multicolumn{2}{|c|}{ CSS-10 } & \multicolumn{2}{|c|}{ CSS-10 } & \multicolumn{2}{|c|}{ CSS-20 } & \multicolumn{2}{|c|}{ CSS-20 } & \multicolumn{2}{|c|}{ CSS-20 } \\
\hline \multicolumn{2}{|c|}{$\mathrm{m}=10, \mathrm{gc}=10$} & \multicolumn{2}{|c|}{$\mathrm{m}=10, \mathrm{gc}=100$} & \multicolumn{2}{|c|}{$\mathrm{m}=10, \mathrm{gc}=1000$} & \multicolumn{2}{|c|}{$\mathrm{m}=20, \mathrm{gc}=10$} & \multicolumn{2}{|c|}{$\mathrm{m}=20, \mathrm{gc}=100$} & \multicolumn{2}{|c|}{$\mathrm{m}=20, \mathrm{gc}=1000$} \\
\hline $\mathrm{f}(\bar{x})$ & $\mathrm{N}$ & $\mathrm{f}(\bar{x})$ & $\mathrm{N}$ & $\mathrm{f}(\bar{x})$ & $\mathrm{N}$ & $\mathrm{f}(\bar{x})$ & $\mathrm{N}$ & $\mathrm{f}(\bar{x})$ & $\mathrm{N}$ & $\mathrm{f}(\bar{x})$ & $\mathrm{N}$ \\
\hline 1.0 & 88 & 0.9 & 53 & 0.9 & 93 & 1.0 & 63 & 0.95 & 40 & 0.9 & 60 \\
\hline 1.0 & 88 & 0.9 & 53 & 0.9 & 93 & 1.0 & 63 & 0.95 & 40 & 0.9 & 60 \\
\hline 0.1 & 1 & 1.0 & 29 & 0.7 & 1 & 1.0 & 63 & 1.0 & 6 & 0.65 & 2 \\
\hline 0.2 & 1 & 0.1 & 7 & 0.9 & 93 & 0.6 & 1 & 0.2 & 2 & 0.05 & 3 \\
\hline 0.3 & 1 & 0.8 & 2 & 0.8 & 1 & 0.1 & 2 & 0.1 & 13 & 0.1 & 8 \\
\hline 0.8 & 1 & 0.3 & 1 & 0.1 & 4 & 0.8 & 4 & 0.4 & 1 & 0.4 & 1 \\
\hline 0.9 & 7 & 0.6 & 1 & & & 0.3 & 1 & 0.85 & 2 & 0.9 & 60 \\
\hline \multirow[t]{2}{*}{0.7} & 1 & 0.2 & 2 & & & 0.85 & 3 & 0.05 & 5 & 0.85 & 14 \\
\hline & & 0.9 & 53 & & & 0.9 & 6 & 0.6 & 1 & 0.8 & 3 \\
\hline \multicolumn{2}{|c|}{$\hat{\mathrm{f}}=0.964$} & \multicolumn{2}{|c|}{$\hat{\mathrm{f}}=0.808$} & \multicolumn{2}{|c|}{$\hat{\mathrm{f}}=0.86$} & \multicolumn{2}{|c|}{$\hat{\mathrm{f}}=0.925$} & \multicolumn{2}{|c|}{$\hat{\mathrm{f}}=0.735$} & \multicolumn{2}{|c|}{$\hat{\mathrm{f}}=0.7475$} \\
\hline
\end{tabular}

The scores specified in Table-11 are obtained by executing a GA with tournament selection and one point crossover on cryptic 3' splice sites for each combination of generation size $(\mathrm{m})$ and generation count $(\mathrm{gc})$. The score function is the confusion score defined in equation-4.

From table-11, we can interpret the results for each generation size(m) and generation count (gc) as follows:

- For a given $\mathrm{m}$, the mean cscore reduces as the number generations increases. This indicates that, as the generations progress, some of the known authentic splice sites 
start appearing in the extrapolated cryptic splice sites.

- For a given $\operatorname{gc}(10,100$, or 1000$)$, the score with the higher generation $\operatorname{size}(\mathrm{m}=20)$ is worse than the lower generation $\operatorname{size}(\mathrm{m}=10)$. For 5' splice sites they are higher. This can be explained by considering the score function in equation-4. The EMR function used in the equation is too rigid for longer sequences. Since 3' splice site sequences are longer than 5' splice sites, we see a different behavior here.

- Based on the frequency of cscore distribution across the hundred executions, $\mathrm{m}=10$ with $\mathrm{gc}=100$ shows the best results. Out of the hundred executions, 93 of them reported a high cscore of 1.0 .

\subsection{Score Summaries}

Table 12: SUMMARY: 5' splice sites | pivot: dataname, metric

\begin{tabular}{|c|c|c|c|c|c|c|}
\hline select & xover & dataname & metric & M & GC & score \\
\hline \hline ROULETTEWHEEL & 2P & AUTH & cscore & 10 & 10 & 1.0 \\
\hline ROULETTEWHEEL & $1 \mathrm{P}$ & AUTH & fitness & 10 & 1000 & 9.64759580424 \\
\hline TOURNAMENT & $1 \mathrm{P}$ & AUTH & pscore & 10 & 100 & 1.0 \\
\hline RANKED & 1P & CSS & cscore & 10 & 1000 & 0.984 \\
\hline ROULETTEWHEEL & UNIFORMORDERBASED & CSS & fitness & 10 & 1000 & 9.83882318289 \\
\hline TOURNAMENT & 1P & CSS & pscore & 10 & 1000 & 1.0 \\
\hline TOURNAMENT & 1P & NEIGHBOR & cscore & 10 & 1000 & 0.995 \\
\hline TOURNAMENT & 1P & NEIGHBOR & fitness & 10 & 1000 & 6.36764544744 \\
\hline ROULETTEWHEEL & UNIFORMORDERBASED & NEIGHBOR & pscore & 10 & 1000 & 0.55 \\
\hline
\end{tabular}


Table 13: SUMMARY: 3' splice sites | pivot: dataname, metric

\begin{tabular}{|c|c|c|c|c|c|c|}
\hline select & xover & dataname & metric & M & GC & score \\
\hline \hline ROULETTEWHEEL & 2P & AUTH & cscore & 10 & 10 & 1.0 \\
\hline ROULETTEWHEEL & UNIFORMORDERBASED & AUTH & fitness & 10 & 10 & 9.84240719451 \\
\hline TOURNAMENT & $1 \mathrm{P}$ & AUTH & pscore & 20 & 1000 & 0.3725 \\
\hline ROULETTEWHEEL & $2 \mathrm{P}$ & CSS & cscore & 10 & 10 & 1.0 \\
\hline TOURNAMENT & $1 \mathrm{P}$ & CSS & fitness & 20 & 10 & 9.66219155445 \\
\hline TOURNAMENT & $1 \mathrm{P}$ & CSS & pscore & 20 & 100 & 0.3715 \\
\hline
\end{tabular}

The summary tables 12 and 13 specify the best scores observed for each combination of dataset (Authentic splice sites, cryptic splice sites, and neighboring splice sites) and score metric (pscore, cscore, and fitness).

We observe that the one-point crossover, roulette wheel selection, and tournament selection dominate the scores for both 5' and 3' splice sites.

Based on these results, it is highly interesting to filter the results and observe only the scores that contribute to the hypothesis the most. Such a point of interest is the cscores for cryptic splice sites that are specified in the next subsection.

\subsubsection{Point of Interest: CSCORE for Cryptic Splice Sites}

In this section, we aggregate the cscores for cryptic splice sites across all combinations of the GA parameters because we want to quantify the intrinsic difference or similarity between authentic and cryptic splice sites. Please note that by the definition of cscore in equation-4, since cryptic and authentic splice sites are competing datasets, it measures the dissimilarity between the datasets based on exact match ratio (equation-1). A high cscore indicates high dissimilarity. 
Table 14: SUMMARY: 5' - BEST GA PARAMETERS FOR CSS CSCORES

\begin{tabular}{|c|c|c|c|c|}
\hline select & xover & M best & GC best & score mean \\
\hline \hline ROULETTEWHEEL & $1 \mathrm{P}$ & 20 & 10 & 0.9665 \\
\hline ROULETTEWHEEL & $2 \mathrm{P}$ & 10 & 10 & 0.935 \\
\hline ROULETTEWHEEL & UNIFORMORDERBASED & 20 & 10 & 0.9105 \\
\hline ROULETTEWHEEL & UNIFORM & 20 & 10 & 0.9475 \\
\hline TOURNAMENT & $1 \mathrm{P}$ & 10 & 10 & 0.361 \\
\hline RANKED & $1 \mathrm{P}$ & 10 & 1000 & 0.984 \\
\hline
\end{tabular}

Table 15: SUMMARY: 3' - BEST GA PARAMETERS FOR CSS CSCORES

\begin{tabular}{|c|c|c|c|c|}
\hline select & xover & M best & GC best & score mean \\
\hline \hline ROULETTEWHEEL & $1 \mathrm{P}$ & \multicolumn{3}{|c|}{ INCONCLUSIVE } \\
\hline ROULETTEWHEEL & $2 \mathrm{P}$ & 20 & 100 & 0.9945 \\
\hline ROULETTEWHEEL & UNIFORMORDERBASED & 10 & 1000 & 0.95 \\
\hline ROULETTEWHEEL & UNIFORM & 10 & 100 & 0.998 \\
\hline TOURNAMENT & $1 \mathrm{P}$ & 10 & 10 & 0.964 \\
\hline RANKED & $1 \mathrm{P}$ & \multicolumn{3}{|c|}{ INCONCLUSIVE } \\
\hline
\end{tabular}

In the tables 15 and 14, we observe that the cscores are high across multiple selection and crossover operators. This proves that the cryptic splice sites are intrinsically different from authentic splice sites.

Although the results presented in this section are promising, the complexity of the GA for obtaining the results should not exceed an exhaustive search on the search space. The next section analyzes the time complexity of the GA for traversing the search space in order to construct the extrapolated 5' and 3' spice sites. 


\section{Analysis}

\section{$7.1 \quad 5$ ' Splice Sites}

The 5' splice sites are represented by a 9-mer with a fixed GT di-nucleotide in positions 4 and 5. Each position in the 9-mer has a cardinality of four corresponding to the four nucleotides - Adenine, Cytosine, Guanine, and Thymine.

Search space size $=4^{9-2}=4^{7}=\mathbf{1 6 3 8 4}$

The PWM-based genetic algorithm described in section 5 extrapolates a subset of this search space. From the results described in table 12 we can see that the algorithm converges decently close to the known splice site data in 1000 generations

Given,

Generation Count $=100$

Generation size $=20$

Number of 9 -mers covered $=20 * 100=2000$

Search space coverage $=2000 / 16384=0.122$

Due to small size of the search space, an exhaustive traversal of the search space is feasible for 5' splice sites. However, by exploring only $12.2 \%$ of the search space, we are able to approximately converge to a subset of the search space that is similar to known splice sites.

\subsection{3' Splice Sites}

The 3' splice sites are represented by a 13-mer with a fixed AG di-nucleotide in positions

11 and 12 . Search space size $=4^{13-2}=4^{1} 1=\mathbf{4 1 9 4 3 0 4}$

Given,

Generation Count $=100$

Generation size $=20$

Number of 9 -mers covered $=20 * 100=2000$

Search space coverage $=2000 / 4194304=0.00047$

Due to the large size of the search space, an exhaustive traversal of the search space is too 
expensive. By exploring only $0.047 \%$ of the search space, we are able to approximately converge to a subset of the search space that is similar to known splice sites. From the results described in section 13 we can see that the algorithm converges decently close to the known splice site data in 100 generations.

\section{Phase 2: Classification Test Using Extrapolated Sets}

In this section, we explore the performance of a classifier trained with the splice sites extrapolated by the GA. The known splice sites are tested with this classifier to measure the accuracy of the model. The main goal is to work around the limitations of the exact match based scoring logic discussed in the section 5.2.6. The classifier-based scoring should outperform the exact match based scores.

\subsection{Classification Primer}

Classification can be defined as : "the task of learning a target function $\mathrm{f}$ that maps each attribute set $\mathrm{x}$ to one of the predefined class labels y." [16]

A Classification model is a set of parameters that are learned using a machine learning technique. The model is the target function that maps any known or unknown attribute set in the input domain space to a class label. The model is also called a classifier.

The task of Machine Learning is defined by Mitchell as follows: "A computer program is said to learn from experience $\mathrm{E}$ with respect to some class of tasks $\mathrm{T}$ and performance measure $\mathrm{P}$ if its performance at tasks in $\mathrm{T}$, as measured by $\mathrm{P}$, improves with experience E."

For example, in the problem of cryptic splice site prediction:

- Task $\mathrm{T}$ is a binary decision of classifying an n-mer as a cryptic splice site or not

- Performance $\mathrm{P}$ is the number of $\mathrm{n}$-mers correctly classified as cryptic splice site / total number of actual cryptic splice sites 
- Experience E is the parsing of known cryptic splice site sequences and known authentic splice site sequences

The classifier is built using machine learning algorithms. The algorithm optimizes a function (for example: minimizing Information Gain in Decision Trees), performs a constrained optimization (maximizing margin in SVM), or performs other optimizations based on input data. The classifier should fit the input data really well and be generic enough to correctly classify unknown data. The problem of fitting input data too well and performing poorly on unknown data is called overfitting. Overfitting is avoided by introducing regularization parameters into the learning process. The opposite of overfitting is underfitting, where the model is so general that it performs poorly on both input data and unknown data. Our main goal is to build a model that performs well on unknown data.

Training algorithms can be regulated using training parameters such as regularization, learning rate, thresholds etc. The model learned during the training phase changes with these parameters. Apart from training itself, it is useful to find the training parameters that generate the best model. This can be achieved by splitting the data into three parts:

- Training data: 60\%; Used in the training phase for current set

- Cross Validation data: 20\%; Used for checking the accuracy for current set of parameters

- Test data: 20\%; Used for checking the accuracy for final set of parameters

Modeling the input data requires a healthy mix of domain knowledge and statistical understanding of the data. It is essential to evaluate the quality of a classifier on the given problem in order to compare the performance of different models. A suitable tool for evaluating the performance of a classifier is the Receiver Operator Characteristic (R.O.C.) curves [17]. 


\subsection{Random Forest Classifiers}

Random Forest(RF) was introduced by Brieman as a bagging-based approach to decision tree classifiers $[18,19]$. Bagging and boosting gained popularity as ensemble-based approaches to machine learning. Both rely on multiple classifiers and weighted voting. AdaBoost aka Adaptive Boosting is a popular boosting technique. The basic idea is that multiple weak classifiers can be combined together to form a strong classifier. In both approaches, the training records are partitioned into $n_{\text {trees }}$ number of bootstrap sets. In boosting, each successive tree is trained on a combination of its own bootstrap set and the misclassified records from its previous tree's bootstrap set. Whereas, in bagging, each bootstrap set is independent. In both approaches, a majority vote is taken across all weak classifiers to classify an unknown record. There are two essential algorithms related to RFs: RF training and error rate estimation.

\section{RF training and testing approach:}

1. Create $n_{\text {tree }}$ sets of randomly selected bootstrap samples.

2. For each bootstrap sample set, randomly draw $m$ predictors out of set of all predictors and train an unpruned decision tree. This generates an ensemble of $n_{\text {tree }}$ decision trees.

3. Use each of the $n_{\text {tree }}$ trees to make $n_{\text {tree }}$ predictions for a test record. Aggregate the result by taking a majority vote.

\section{Error Rate estimation:}

1. Create an Out Of Bag (OOB) samples set i.e. the samples not in bootstrap, for each of the $n_{\text {tree }}$ bootstrap sets.

2. Use the $\mathrm{OOB}$ data to make predictions by majority vote. Aggregate the results to compute OOB error rate of the Random Forest.

\section{Random Forest parameters:}

For a high number of predictors, one should generate as many trees as computationally 
feasible. This is required to ensure that the selection of each predictor is equiprobable. Ideally, this approach should lead to similar results across multiple executions of random forest induction.

Other parameters include number of candidate predictors selected in each tree, size of each tree, and resampling scheme or the bootstrap sample selection scheme for each iteration [18].

Most of the parameters can be estimated based on OOB frequency . The number of trees should be as large as possible. However, the optimal values of the parameters are dependent on the data itself. One should generate a random forests for all combinations of parameters and select the parameters that give the RF with minimum $\mathrm{OOB}$ error frequency [18].

\subsection{Modeling the Random Forest Classifier}

In terms of the notations defined in section-5.2, we form two tables as follows:

$$
\begin{gathered}
R_{\text {train }}=E A_{5} \cup E C_{5} \cup E N_{5} \\
\text { RFtest }_{5}=A_{5} \cup C_{5} \cup N_{5}
\end{gathered}
$$

The GA setting with 1000 generation that reports the maximum mean fitness is chosen. The best chromosome from each of the 1000 generations is added to the training data. This process is repeated for each authentic, cryptic, and neighboring data.

A new column is added to the tables to indicate the labels as follows: For RFtrain R :

$$
\begin{aligned}
& \operatorname{label}(\mathrm{x})=0 \forall x \in\left\{E A_{5}\right\} \\
& \operatorname{label}(\mathrm{x})=1 \forall x \in\left\{E C_{5}\right\} \\
& \operatorname{label}(\mathrm{x})=2 \forall x \in\left\{E N_{5}\right\}
\end{aligned}
$$


For RFtest :

$$
\begin{aligned}
& \operatorname{label}(\mathrm{x})=0 \forall x \in\left\{A_{5}\right\} \\
& \operatorname{label}(\mathrm{x})=1 \forall x \in\left\{C_{5}\right\} \\
& \operatorname{label}(\mathrm{x})=2 \forall x \in\left\{N_{5}\right\}
\end{aligned}
$$

As shown in figure-17, the RFtrain is $_{5}$ used as training data to train a Random Forest classifier. The accuracy of the model is tested with the RFtest ${ }_{5}$ data.

A high accuracy indicates that the training data closely captures the structure of the actual known splice sites. The training data is extrapolated from GA from a random initial population and PWM models of the known splice sites. 


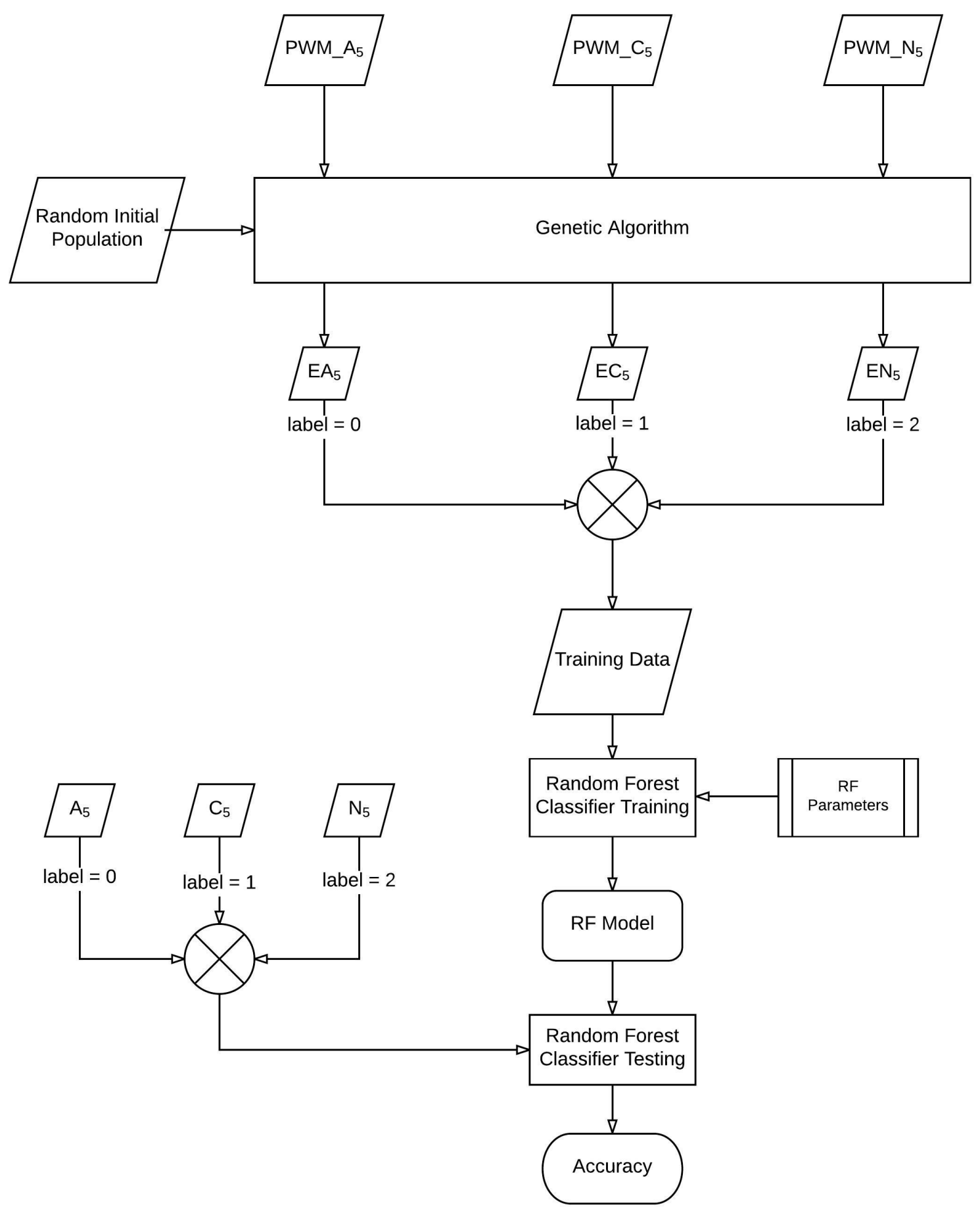

Figure 17: Random Forest Classifier workflow for extrapolated splice sites 


\section{Conclusion}

The results obtained in section 6 show that the mean fitness increases with generation count. This indicates that GA is successfully traversing the search space in a controlled fashion. GA converges much faster than an exhaustive search space traversal. This becomes more evident in the analysis of 3' splice sites, which contain longer 13-mer splice site sequences.

The high similarity scores (pscore) in the summary tables 13 and 12 indicate a high similarity between the extrapolated set and the corresponding training splice site data. Similarly, the high dissimilarity scores (cscore) indicate a high dissimilarity between the extrapolated set and the corresponding competing (section 5.2.4) splice site data. These observations provide us the evidence required to confirm the hypothesis that the competing splice sites are intrinsically dissimilar.

The scores for the 3' splice sites are worse than the 5' splice sites. This is explained in section 5.2.6. The scores for the 3' splice sites can be improved by using local alignmentbased algorithms for computing the scores because they are longer sequences than 5' splice sites.

We also observed that the roulette wheel selection, tournament selection, and one point crossover give us most of the best scores across all combinations of GA parameters.

Another interesting fact is that we get high scores in GA when we use PWM scores as fitness for the splice site datasets. The Position Weight Matrix is known to be a good probabilistic model for splice sites in nucleotide sequences [15].

Some of the topics not covered here are schema theorem and analysis, representations and encoding techniques, and alternative random number generators. Schema analysis gives a more detailed understanding of the behavior of the GA. It is also used to compute a suitable crossover probability. Also, it has been shown that variations in the random number generator implementation have an impact on the performance of the GA. 


\section{References}

[1] Ezkurdia, I., Juan, D., Rodriguez, J. M., Frankish, A., Diekhans, M., Harrow, J., ... \& Tress, M. L. (2014). Multiple evidence strands suggest that there may be as few as 19000 human protein-coding genes. Human molecular genetics, 23(22), 5866-5878. Available [Online]: https://www.ncbi.nlm.nih.gov/pmc/articles/PMC4204768/ Last retrieved: $05 / 21 / 2017$

[2] Pollastro, P., \& Rampone, S. (2003). HS3D: homosapiens splice site data set. Nucleic Acids Research, (Annual Database). Available [Online]: http://www.sci. unisannio.it/docenti/rampone/ Last retrieved: 04/26/2017

[3] Pollastro, P., \& Rampone, S. (2002). HS3D, a dataset of Homo Sapiens splice regions, and its extraction procedure from a major public database. International Journal of Modern Physics C, 13(08), 1105-111\%. Available [Online]: https://www . researchgate.net/profile/Salvatore_Rampone/publication/2549884_HS3D_a_ Dataset_of_Homo_Sapiens_Splice_Regions_and_its_Extraction_Procedure_ from_a_Major_Public_Database/links/0912f510aade936928000000.pdf Last retrieved: $04 / 26 / 2017$

[4] DBASS: Database of Aberrant splice sites in human disease genes Available [Online]: http: //www.dbass.org.uk/ Last retrieved: 04/26/2017

[5] Vorechovsky, I. (2006). Aberrant 3' splice sites in human disease genes: mutation pattern, nucleotide structure and comparison of computational tools that predict their utilization. Nucleic acids research, 34(16), 4630-4641. Available [Online]: https://academic.oup.com/nar/article/34/16/4630/3111901/ Aberrant-3-splice-sites-in-human-disease-genes Last retrieved: 04/26/2017

[6] Kralovicova, J., Christensen, M. B., \& Vorechovsky, I. (2005). Biased exon/intron distribution of cryptic and de novo 3' splice sites. Nucleic acids research, 33(15), 48824898. Available [Online]: https://academic.oup.com/nar/article/33/15/4882/ 
2401079/Biased-exon-intron-distribution-of-cryptic-and-de Last retrieved: $04 / 26 / 2017$

[7] Buratti, E., Chivers, M., Kralovicova, J., Romano, M., Baralle, M., Krainer, A. R., \& Vorechovsky, I. (2007). Aberrant 5' splice sites in human disease genes: mutation pattern, nucleotide structure and comparison of computational tools that predict their utilization. Nucleic acids research, 35(13), 4250-4263. Available [Online]: https://academic.oup.com/nar/article/35/13/4250/1203203/ Aberrant-5-splice-sites-in-human-disease-genes Last retrieved: 04/26/2017

[8] De Jong, K., Fogel, D., \& Schwefel, H. P. (1997). Handbook of Evolutionary Computation. IOP Publishing Ltd and Oxford University Press. Available [Online]: http://citeseerx.ist.psu.edu/viewdoc/download?doi=10.1.1.375. 6494\&rep=rep1\&type=pdf Last retrieved: 04/26/2017

[9] Sastry, K., Goldberg, D. E., \& Kendall, G. (2014). Genetic algorithms. In Search methodologies (pp. 93-117). Springer US. Available [Online]: http://www. graham-kendall.com/papers/sgk2014.pdf Last retrieved: 04/26/2017

[10] Goldberg, D. E., \& Holland, J. H. (1988). Genetic algorithms and machine learning. Machine learning, 3(2), 95-99.

[11] Back, T., \& Khuri, S. (1994, June). An evolutionary heuristic for the maximum independent set problem. In Evolutionary Computation, 1994. IEEE World Congress on Computational Intelligence., Proceedings of the First IEEE Conference on (pp. 531-535). IEEE. Available [Online]: https://pdfs.semanticscholar.org/6881/ 7937ebf74d20c68e04ecc9b4aa04a4e8d8dc.pdf Last retrieved: 04/26/2017

[12] Khuri, S. (2012). Lecture Notes on Genetic Algorithms

[13] Khuri, S. (2017). Lecture Notes on BioInformatics

[14] Xia, X. (2012). Position weight matrix, gibbs sampler, and the associated significance tests in motif characterization and prediction. Scientifica, 2012. Available [On- 
line]: http://downloads.hindawi.com/journals/scientifica/2012/917540.pdf Last retrieved: $04 / 26 / 2017$

[15] Claverie, J. M., \& Audic, S. (1996). The statistical significance of nucleotide position-weight matrix matches. Computer applications in the biosciences: CABIOS, 12(5), 431-439. Available [Online]: http://bioinformatics.oxfordjournals.org/ content/12/5/431.full.pdf Last retrieved: 04/26/2017

[16] Pang-Ning, T., Steinbach, M., \& Kumar, V. (2006). Introduction to data mining. In Library of congress (Vol. 74)

[17] Davis, J., \& Goadrich, M. (2006, June). The relationship between Precision-Recall and ROC curves. In Proceedings of the 23rd international conference on Machine learning (pp. 233-240). ACM. Available [Online]: http://machinelearning.wustl. edu/mlpapers/paper_files/icml2006_DavisG06.pdf Last retrieved: 04/26/2017

[18] Boulesteix, A. L., Janitza, S., Kruppa, J., \& König, I. R. (2012). Overview of random forest methodology and practical guidance with emphasis on computational biology and bioinformatics. Wiley Interdisciplinary Reviews: Data Mining and Knowledge Discovery, 2(6), 493-50\%. Available [Online]: https://epub.ub.uni-muenchen.de/ 13766/1/TR.pdf Last retrieved: 04/26/2017

[19] Liaw, A., \& Wiener, M. (2002). Classification and regression by randomForest. $R$ news, 2(3), 18-22. Available [Online]: http://ai2-s2-pdfs.s3.amazonaws.com/ 6e63/3b41d93051375ef9135102d54fa097dc8cf8.pdf Last retrieved: 04/26/2017 